\title{
Calcium Homeostasis in a Local/Global Whole Cell Model of Permeabilized Ventricular Myocytes with a Langevin Description of Stochastic Calcium Release
}

\author{
Xiao Wang \\ Seth H. Weinberg \\ Gregory D. Smith \\ William \& Mary, gdsmit@wm.edu \\ Yan Hao \\ Eric A. Sobie
}

Follow this and additional works at: https://scholarworks.wm.edu/aspubs

Part of the Applied Mathematics Commons

\section{Recommended Citation}

Wang, Xiao; Weinberg, Seth H.; Smith, Gregory D.; Hao, Yan; and Sobie, Eric A., Calcium Homeostasis in a Local/Global Whole Cell Model of Permeabilized Ventricular Myocytes with a Langevin Description of Stochastic Calcium Release (2015). American Journal of Physiology-Heart and Circulatory Physiology, 308(5), H510-H523.

https://doi.org/10.1152/ajpheart.00296.2014

This Article is brought to you for free and open access by the Arts and Sciences at W\&M ScholarWorks. It has been accepted for inclusion in Arts \& Sciences Articles by an authorized administrator of W\&M ScholarWorks. For more information, please contact scholarworks@wm.edu. 


\title{
Calcium homeostasis in a local/global whole cell model of permeabilized ventricular myocytes with a Langevin description of stochastic calcium
} release

\author{
Xiao Wang, ${ }^{1}$ Seth H. Weinberg, ${ }^{1}$ Yan Hao, ${ }^{2}$ Eric A. Sobie, ${ }^{3}$ and Gregory D. Smith ${ }^{1}$ \\ ${ }^{1}$ Department of Applied Science, The College of William and Mary, Williamsburg, Virginia; ${ }^{2}$ Department of Mathematics \\ and Computer Science, The Hobart and William Smith Colleges, Geneva, New York; and ${ }^{3}$ Department of Pharmacology \\ and Systems Therapeutics, Mount Sinai School of Medicine, New York, New York
}

Submitted 2 May 2014; accepted in final form 4 December 2014

Wang X, Weinberg SH, Hao Y, Sobie EA, Smith GD. Calcium homeostasis in a local/global whole cell model of permeabilized ventricular myocytes with a Langevin description of stochastic calcium release. Am J Physiol Heart Circ Physiol 308: H510-H523, 2015. First published December 5, 2014; doi:10.1152/ajpheart.00296.2014.Population density approaches to modeling local control of $\mathrm{Ca}^{2+}$ induced $\mathrm{Ca}^{2+}$ release in cardiac myocytes can be used to construct minimal whole cell models that accurately represent heterogeneous local $\mathrm{Ca}^{2+}$ signals. Unfortunately, the computational complexity of such "local/global" whole cell models scales with the number of $\mathrm{Ca}^{2+}$ release unit (CaRU) states, which is a rapidly increasing function of the number of ryanodine receptors (RyRs) per CaRU. Here we present an alternative approach based on a Langevin description of the collective gating of RyRs coupled by local $\mathrm{Ca}^{2+}$ concentration $\left(\left[\mathrm{Ca}^{2+}\right]\right)$. The computational efficiency of this approach no longer depends on the number of RyRs per CaRU. When the RyR model is minimal, Langevin equations may be replaced by a single FokkerPlanck equation, yielding an extremely compact and efficient local/ global whole cell model that reproduces and helps interpret recent experiments that investigate $\mathrm{Ca}^{2+}$ homeostasis in permeabilized ventricular myocytes. Our calculations show that elevated myoplasmic $\left[\mathrm{Ca}^{2+}\right]$ promotes elevated network sarcoplasmic reticulum (SR) $\left[\mathrm{Ca}^{2+}\right]$ via SR $\mathrm{Ca}^{2+}$-ATPase-mediated $\mathrm{Ca}^{2+}$ uptake. However, elevated myoplasmic $\left[\mathrm{Ca}^{2+}\right]$ may also activate RyRs and promote stochastic SR $\mathrm{Ca}^{2+}$ release, which can in turn decrease $\mathrm{SR}\left[\mathrm{Ca}^{2+}\right]$. Increasing myoplasmic $\left[\mathrm{Ca}^{2+}\right]$ results in an exponential increase in spark-mediated release and a linear increase in nonspark-mediated release, consistent with recent experiments. The model exhibits two steady-state release fluxes for the same network SR $\left[\mathrm{Ca}^{2+}\right]$ depending on whether myoplasmic $\left[\mathrm{Ca}^{2+}\right]$ is low or high. In the later case, spontaneous release decreases SR $\left[\mathrm{Ca}^{2+}\right]$ in a manner that maintains robust $\mathrm{Ca}^{2+}$ sparks.

Langevin equation; Fokker-Planck equation; calcium release site; multiscale whole cell model; calcium homeostasis

INTRACELLULAR CALCIUM $\left(\mathrm{Ca}^{2+}\right)$ signaling involves a complex interplay between global (cell-wide) changes in $\mathrm{Ca}^{2+}$ concentration $\left(\left[\mathrm{Ca}^{2+}\right]\right)$ and local (subcellular) $\mathrm{Ca}^{2+}$ release events. Local signals are caused by plasma membrane $\mathrm{Ca}^{2+}$ influx and release of $\mathrm{Ca}^{2+}$ from intracellular stores, primarily the endoplasmic/sarcoplasmic reticulum (ER/SR). Spatially localized $\mathrm{Ca}^{2+}$ release events mediated by clusters of intracellular $\mathrm{Ca}^{2+}$ channels, $\mathrm{IP}_{3}$ receptors $\left(\mathrm{IP}_{3} \mathrm{Rs}\right)$ or ryanodine receptors (RyRs) on the ER/SR membrane, are referred to as " $\mathrm{Ca}^{2+}$ sparks" or "puffs" (see Ref. 2 for review).

Address for reprint requests and other correspondence: G. D. Smith, Dept. of Applied Science, McGlothlin-Street Hall, Rm. 305, The College of William and Mary, Williamsburg, VA 23187 (e-mail: greg@as.wm.edu).
While plasma membrane ion channels in a small cell experience essentially the same time course of membrane voltage, intracellular $\mathrm{Ca}^{2+}$ channels experience radically different local $\left[\mathrm{Ca}^{2+}\right]$, even during global $\mathrm{Ca}^{2+}$ responses, and clusters of $\mathrm{IP}_{3} \mathrm{Rs}$ and RyRs are in fact only locally coupled via the buffered diffusion of intracellular $\mathrm{Ca}^{2+}$. That is, when one or several of the channels in a $\mathrm{Ca}^{2+}$ release unit (CaRU) are open, the $\left[\mathrm{Ca}^{2+}\right]$ experienced by spatially localized channels is dramatically different from the $\left[\mathrm{Ca}^{2+}\right]$ in the bulk myoplasm. For this reason, conventional whole cell modeling of $\mathrm{Ca}^{2+}$ dynamics based on Hodgkin-Huxley-like gating variables for the dynamics of intracellular channels is not always appropriate.

Mechanistic models of ER/SR $\mathrm{Ca}^{2+}$ release often represent the stochastic gating of $\mathrm{Ca}^{2+}$ channels using Monte Carlo methods. When these approaches are applied to cardiac myocytes, voltage-gated L-type $\mathrm{Ca}^{2+}$ channel(s) interact with a cluster of RyRs through changes in $\left[\mathrm{Ca}^{2+}\right]$ in small "dyadic subspaces" between the sarcolemmal and SR membranes. These models also sometimes consider depletion of junctional SR $\left[\mathrm{Ca}^{2+}\right]$ that may influence $\mathrm{Ca}^{2+}$ spark termination and refractoriness $(31,32,35)$. Realistic global (cell-wide) SR $\mathrm{Ca}^{2+}$ release can be reproduced by Monte Carlo simulation of the stochastic triggering of sparks from hundreds to thousands of CaRUs $(19,20,29,32)$. However, such simulations of local control of excitation-contraction coupling are computationally demanding, especially when each CaRU is composed of interacting Markov chain models of individual RyRs (e.g., see Ref. 23).

Population density approaches are an alternative to Monte Carlo simulations that produce realistic and computationally efficient models by using a master equation to represent heterogeneous local $\mathrm{Ca}^{2+}$ signals in dyadic subspaces and junctional SR domains (37). This approach involves the numerical solution of advection-reaction equations for the time-dependent bivariate probability density of subspace and junctional SR $\left[\mathrm{Ca}^{2+}\right]$ conditioned on CaRU state, coupled to ordinary differential equations (ODEs) for the bulk myoplasmic and network SR $\left[\mathrm{Ca}^{2+}\right]$. This methodology was validated in prior work (37) and an associated moment-based approach to simulating the probability distribution of junctional SR $\left[\mathrm{Ca}^{2+}\right]$ was benchmarked to be several orders of magnitude faster than conventional Monte Carlo simulation of the dynamics of local $\mathrm{Ca}^{2+}$ associated with a physiological number of CaRUs (38).

One disadvantage of the population density approaches to modeling local control is that their run times (computational efficiency) are proportional to the number of CaRU states. When realistically modeled as the collective gating of identical 
and indistinguishable RyRs, the number of CaRU states is exponential in the number of channel states. Population density and moment-based methods for multiscale (i.e., local/global) whole cell modeling are limited by this state-space explosion.

Here we present an alternative local/global whole cell modeling approach based on a Langevin formulation of the stochastic $\mathrm{Ca}^{2+}$ release via CaRUs. We assume that the number of RyRs per CaRU is large enough that the fraction of channels in each state can be treated as a continuous variable. We show that the Langevin description of the collective gating of RyRs is a good approximation to the corresponding discrete-state continuous-time Markov chain model when the number of RyRs per release site is in the physiological range. By coupling the numerical solution of such Langevin equations to balance equations for the bulk myoplasmic and network SR $\left[\mathrm{Ca}^{2+}\right]$, a local/global whole cell model is produced whose run time scales with the number of states in the Markov chain model for an individual RyR, as opposed to the far greater number of states in a compositionally defined CaRU. When the RyR model is minimal, these Langevin equations may be replaced by a single Fokker-Planck equation for a randomly sampled CaRU, yielding an extremely compact and efficient local/ global whole cell model. We illustrate the usefulness and computational efficiency of the Fokker-Planck equation-based local/global whole cell model by performing parameter studies motivated by recent experiments $(3,40)$.

In intact ventricular myocytes of the healthy heart, the balance of diastolic SR $\mathrm{Ca}^{2+}$ leak and uptake maintains the appropriate $\mathrm{SR} \mathrm{Ca}^{2+}$ load. While the SR $\mathrm{Ca}^{2+}$ leak is mediated primarily by RyRs, the contributions of spark- and nonsparkmediated SR $\mathrm{Ca}^{2+}$ release depend on the concentration of both myoplasmic and SR $\left[\mathrm{Ca}^{2+}\right](10,27,30,40)$. When SR $\left[\mathrm{Ca}^{2+}\right]$ is low, $\mathrm{SR} \mathrm{Ca}^{2+}$ leak occurs primarily through spark-independent pathways. Conversely, when SR $\left[\mathrm{Ca}^{2+}\right]$ is high, spontaneous $\mathrm{Ca}^{2+}$ sparks make a large contribution to SR leak. In pathophysiological conditions that include $\mathrm{SR} \mathrm{Ca}^{2+}$ overload, increased SR $\mathrm{Ca}^{2+}$ leak may generate spontaneous sparks that trigger $\mathrm{Ca}^{2+}$-induced $\mathrm{Ca}^{2+}$ release (CICR) from neighboring CaRUs, thereby initiating arrhythmogenic spontaneous $\mathrm{Ca}^{2+}$ waves (9).

With the use of permeabilized ventricular myocytes, a reduced experimental preparation that allows precise control of myoplasic $\left[\mathrm{Ca}^{2+}\right]$, Bovo et al. (3) observed that increasing myoplasmic $\left[\mathrm{Ca}^{2+}\right]$ results in an exponential increase in sparkmediated release and a linear increase in nonspark-mediated release. These results are reproduced by the Fokker-Planck equation-based local/global whole cell model that is the focus of this article. In addition, the model predicts potentially significant characteristics of $\mathrm{Ca}^{2+}$ homeostasis in permeabilized cells. For example, in the local/global whole cell model, two distinct steady states may exist for a given network SR $\left[\mathrm{Ca}^{2+}\right]$. One steady-state corresponds to low myoplasmic $\left[\mathrm{Ca}^{2+}\right]$ and small SR $\mathrm{Ca}^{2+}$ release flux that is dominated by stochastic leak, while the other corresponds to high myoplasmic $\left[\mathrm{Ca}^{2+}\right]$ and large release flux mediated by $\mathrm{Ca}^{2+}$ sparks. Interestingly, for any clamped myoplasmic $\left[\mathrm{Ca}^{2+}\right]$ that is large enough to trigger spark-mediated release, the local/global model predicts that the resulting spontaneous stochastic $\mathrm{Ca}^{2+}$ release tends to decrease the network $\mathrm{SR} \mathrm{Ca}^{2+}$ load just enough to maintain robust $\mathrm{Ca}^{2+}$ sparks.

\section{METHODS}

Markov chain description of a $\mathrm{Ca}^{2+}$ release site. The most straightforward starting point for the presentation of the Langevin description of a $\mathrm{Ca}^{2+}$ release site (CaRU) is the following two-state Markov chain model of a stochastically gating RyR,

$$
\text { (closed) } \mathrm{C} \underset{k^{-}}{\stackrel{k^{+} \mathrm{c}^{\eta}}{\rightleftarrows}} \mathrm{O} \text { (open), }
$$

where $\mathrm{c}$ is the local $\left[\mathrm{Ca}^{2+}\right], k^{+} \mathrm{c}^{\eta}$ and $k^{-}$are transition rates with units of reciprocal time, $k^{+}$is an association rate constant with units of concentration $^{-}$.time $^{-1}$, and $\eta$ is the cooperativity of $\mathrm{Ca}^{2+}$ binding. Under the assumption that a collection of $N$ two-state RyRs are instantaneously coupled by a local $\left[\mathrm{Ca}^{2}\right]$ associated with the RyR cluster, the transition diagram for the CaRU as a collective entity is (8)

$$
0 \underset{k^{-}}{\stackrel{N k^{+} c_{0}^{\eta}}{\rightleftarrows}} 1 \underset{2 k^{-}}{\stackrel{(N-1) k^{+} c_{1}^{\eta}}{\rightleftarrows}} \ldots \underset{(N-1) k^{-}}{\stackrel{2 k^{+} c_{N-2}^{\eta}}{\rightleftarrows}} N-1 \underset{N k^{-}}{\stackrel{k^{+} c_{N-1}^{\eta}}{\rightleftarrows}} N,
$$

where the states $\{0,1, \ldots, N\}$ correspond to the number of open channels $\left(N_{\mathrm{O}}\right)$ and $\mathrm{c}_{n}$ is the local $\left[\mathrm{Ca}^{2+}\right]$ experienced by RyRs when $N_{\mathrm{O}}=n$.

Figure $1 A$ shows a Markov chain simulation of a CaRU composed of $N=20$ two-state channels. For simplicity we here assume that the local $\left[\mathrm{Ca}^{2+}\right]$ is a linear function of $N_{\mathrm{O}}$, that is,

$$
\mathrm{c}_{n}=\mathrm{c}_{\infty}+n \mathrm{c}_{*},
$$

where $\mathrm{c}_{\infty}$ is the bulk or background $\left[\mathrm{Ca}^{2+}\right]$ and $\mathrm{c}_{*}$ determines the increment in local $\left[\mathrm{Ca}^{2+}\right]$ following an individual RyR opening. The corresponding relationship between $N_{\mathrm{O}}$ and local $\left[\mathrm{Ca}^{2+}\right]$ is more realistic in the local/global whole cell model (Eqs. 24 and 25).

Langevin $\mathrm{Ca}^{2+}$ release site model. We will write $f_{\mathrm{O}}(t)$ as the time-varying fraction of open RyRs, that is,

$$
f_{\mathrm{O}}(t)=\frac{N_{\mathrm{O}}(t)}{N} \text {. }
$$

The Langevin equation that corresponds to a CaRU composed of $N$ two-state channels (see above) is a stochastic ordinary differential equation $(\mathrm{SDE})$ of the form

$$
\frac{\mathrm{d} f_{\mathrm{O}}}{\mathrm{d} t}=k^{+}\left(\mathrm{c}_{\infty}+\overline{\mathrm{c}} f_{\mathrm{O}}\right)^{\eta}\left(1-f_{\mathrm{O}}\right)-k^{-} f_{\mathrm{O}}+\xi(t),
$$

where $\overline{\mathrm{c}}=N \mathrm{c}_{*}$ and $\xi(t)$ is a rapidly varying forcing term (Gaussian white noise) with zero mean

$$
\langle\xi(t)\rangle=0 .
$$

As discussed in APPENDIX A, the magnitude of the noise term, $\xi(t)$, is characterized by the two-time covariance $(16,26)$,

$$
\left\langle\xi(t) \xi\left(t^{\prime}\right)\right\rangle=\gamma\left(f_{\mathrm{O}}\right) \delta\left(t-t^{\prime}\right)
$$

where $\delta$ is the Dirac delta function and $\gamma\left(f_{\mathrm{O}}\right)$ is the infinitesimal variance of $f_{\mathrm{O}}$ and is given by

$$
\gamma\left(f_{\mathrm{O}}\right)=\frac{k^{+}\left(\mathrm{c}_{\infty}+\overline{\mathrm{c}} f_{\mathrm{O}}\right)^{\eta}\left(1-f_{\mathrm{O}}\right)+k^{-} f_{\mathrm{O}}}{N} .
$$

With the use of parameters that lead to $\mathrm{Ca}^{2+}$ sparks, Fig. $1 B$ shows that the Langevin simulation of a 20 -channel CaRU is qualitatively similar to the corresponding Markov chain simulation (Fig. 1A).

Equivalence of Markov chain and Langevin formulations. The Langevin CaRU model is expected to well approximate the Markov chain model when the number of RyRs per CaRU $(N)$ is sufficiently large. To determine whether this convergence occurs for a physiological number of RyRs (10-200 per CaRU in skeletal and cardiac myocytes; Ref. 11), we compare the stationary distributions for $N_{\mathrm{O}}$ 
Fig. 1. Comparison and agreement of the $\mathrm{Ca}^{2+}$ release site model using Markov chain and Langevin formulation. A: Markov chain simulation of 20 two-state ryanodine receptors (RyRs) coupled via local $\mathrm{Ca}^{2+}$ concentration $\left(\left[\mathrm{Ca}^{2+}\right]\right)(E q .2)$ using Gillespie's stochastic tion obtained by numerically integrating Eqs. 5-8 using the Euler-Maruyama method (15). $C$ : stationary distribution of the Markov chain (calculated analytically, Eq. 2, white histogram) and the binned (discretized) probability distribution for the Langevin formulation (calculated from simulations, black histogram) and Fokker-Planck equation (calculated analytically, Eq. $14,+$ symbols). $D$ : stationary distribution of Fokker-Planck equation (Eqs. 14 and 15). Parameters are chosen to illustrate the phenomenon of stochastic $\mathrm{Ca}^{2+}$ excitability: $k^{+}=0.15 \mu \mathrm{M}^{-\eta} \cdot \mathrm{ms}^{-1}, k^{-}=0.05 \mathrm{~ms}^{-1}, \mathrm{c}_{*}=$ $0.06 \mu \mathrm{M}, \eta=2$. simulation algorithm (17). $B$ : Langevin simula-
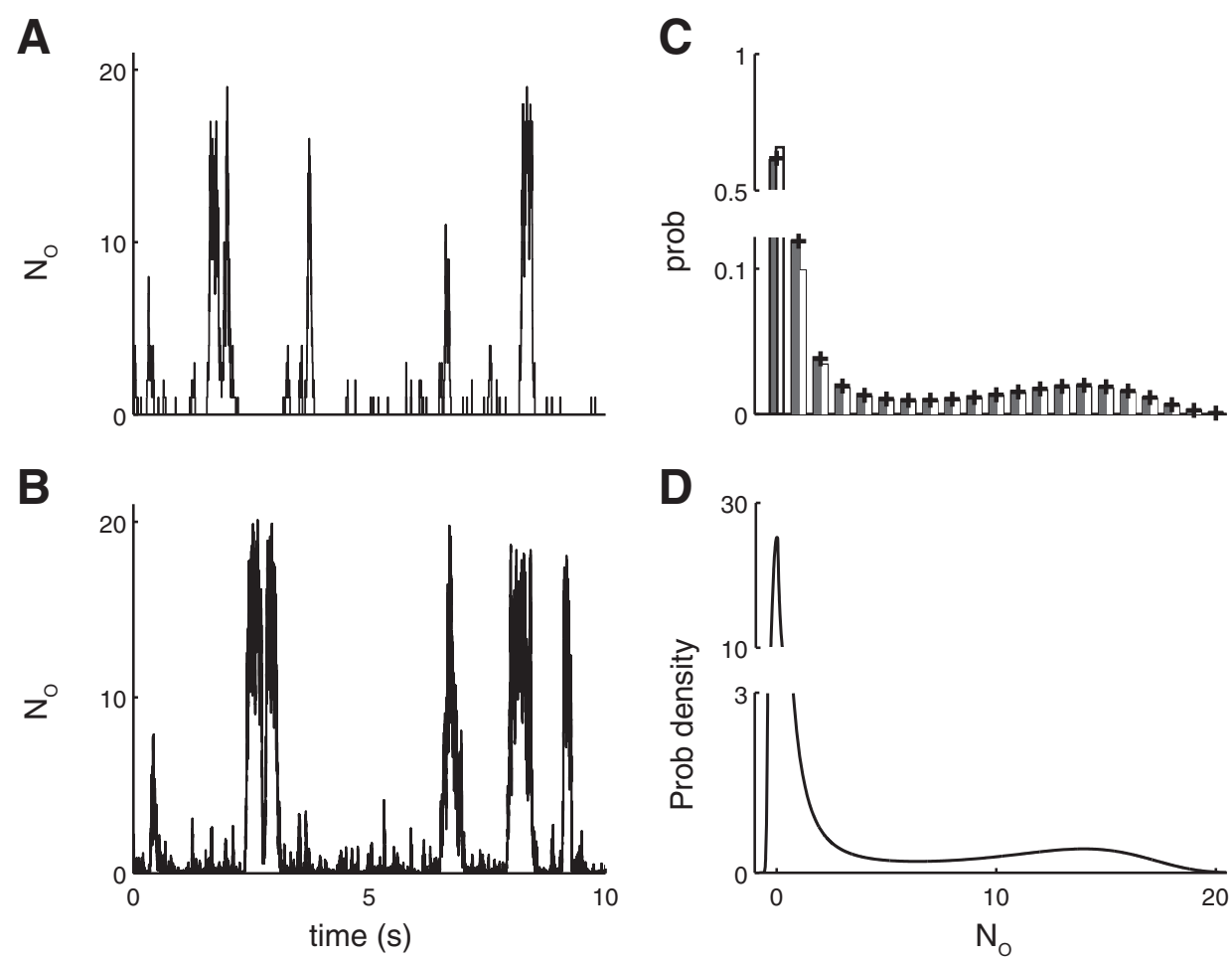

(Fig. $1 C$ ). The white histogram of Fig. $1 C$ shows that the bimodal Markov chain stationary distribution has a local maxima near $N_{\mathrm{O}}=0$ and $N_{\mathrm{O}}=15$. This distribution reflects the fact that the $N$ RyRs are usually closed but occasionally open in concert as is characteristic for $\mathrm{Ca}^{2+}$ sparks. Comparison to the corresponding distribution of the Langevin model (black histogram) shows that the SDE formulation is a good approximation to the Markov chain, even when the number of RyRs per CaRU is on the low end of the physiological range.

In the Langevin CaRU formulation, the state space for $f_{\mathrm{O}}$ is continuous $\left(0 \leq f_{\mathrm{O}} \leq 1\right)$. The Fokker-Planck equation solved by the probability density function for the fraction of open channels, $\rho(f, t)$, is given by (12)

$$
\frac{\partial \rho}{\partial t}=-\frac{\partial}{\partial f}[\alpha \rho]+\frac{1}{2} \frac{\partial^{2}}{\partial f^{2}}[\gamma \rho]
$$

where $\rho(f, t) \mathrm{d} f=\operatorname{Pr}\left\{f \leq f_{\mathrm{O}}(t)<f+\mathrm{d} f\right\}$. Note that in these expressions, $f_{\mathrm{O}}$ is the random variable and $f$ is the independent variable of the probability density. The drift and diffusion terms in Eq. 9 are given by

$$
\begin{gathered}
\alpha(f)=v^{+}-v^{-}, \\
\gamma(f)=\left(v^{+}+v^{-}\right) / N,
\end{gathered}
$$

where $v^{ \pm}(f)$ are the rates of the elementary processes leading to an increase and decrease in the fraction of open channels, that is,

$$
\begin{aligned}
v^{+}(f)= & k^{+}\left(\mathrm{c}_{\infty}+\overline{\mathrm{c}} f\right)^{\eta}(1-f), \\
& v^{-}(f)=k^{-} f,
\end{aligned}
$$

and $\overline{\mathrm{c}}=\mathrm{c} * N$ as above (Eq. 5).

Setting the left-hand side of $E q .9$ equal to zero $(\partial \rho / \partial t=0)$, denoting the stationary density by $\rho_{\mathrm{ss}}(f)$ and applying boundary conditions $\rho_{\mathrm{ss}}(f) \rightarrow 0$ as $f \rightarrow \pm \infty$, it can be shown that (12)

$$
\rho_{\mathrm{ss}}(f)=\frac{\theta}{\gamma} \exp \{2 U\}
$$

where $\theta$ is a normalization constant such that $\int \rho_{\mathrm{ss}}(f) \mathrm{d} f=1$ and

$$
U(f)=\int_{\mathrm{a}}^{f} \frac{\alpha\left(f^{\prime}\right)}{\gamma\left(f^{\prime}\right)} \mathrm{d} f^{\prime}
$$

is an accumulation function with a lower limit of integration satisfying $\alpha(f) / \gamma(f)=0$ for $f \leq a$. In fact, $U$ may be any antiderivative satisfying $U^{\prime}=\alpha / \gamma$, because the normalization of $\rho_{\mathrm{ss}}$ determines the constant of integration.

Figure $1 D$ shows the stationary density $\rho_{\mathrm{ss}}(f)$ for the 20 -channel Fokker-Planck CaRU model described above. The + symbols in Fig. $1 C$ are binned values of $\rho_{\mathrm{ss}}(f)$ that may be compared with (and agree with) the stationary distributions of the Markov chain (white histogram) and Langevin (black histogram) descriptions. APPENDIX B provides more comparisons of Markov chain, Langevin, and FokkerPlanck CaRU simulations.

Full local/global whole cell model. Having validated the Langevin CaRU model in the previous sections, we are prepared to construct the local/global whole cell model of $\mathrm{Ca}^{2+}$ homeostasis in permeabilized ventricular myocytes that is the focus of this article. Figure 2 shows the relationship between the bulk $\mathrm{Ca}^{2+}$ concentrations of the myoplasm $\left(\mathrm{c}_{\text {myo }}\right)$ and the network SR $\left(\mathrm{c}_{\mathrm{nsr}}\right)$ and the local $\mathrm{Ca}^{2+}$ concentrations associated with each CaRU. With respect to global aspects of $\mathrm{Ca}^{2+}$ signaling, the material balance equations of the whole cell model are

$$
\begin{aligned}
& \frac{\mathrm{dc}_{\mathrm{myo}}}{\mathrm{d} t}=J_{\text {myo }}^{\mathrm{T}}-J_{\text {pump }}+J_{\mathrm{pm}} \\
& \frac{\mathrm{dc}_{\mathrm{nsr}}}{\mathrm{d} t}=\frac{1}{\Lambda_{\mathrm{nsr}}}\left(-J_{\mathrm{nsr}}^{\mathrm{T}}+J_{\text {pump }}\right)
\end{aligned}
$$

where $\Lambda_{\text {nsr }}$ is an effective volume ratio that accounts for both physical volume and $\mathrm{Ca}^{2+}$ buffering capacity of the myoplasm and network $\mathrm{SR}$. A plasma membrane flux may take the form $J_{\mathrm{pm}}=k_{\mathrm{pm}}\left(\mathrm{c}_{\mathrm{ext}}-\right.$ $\mathrm{c}_{\text {myo }}$ ). The sarco(endo)plasmic reticulum $\mathrm{Ca}^{2+}$-ATPase (SERCA) type $\mathrm{Ca}^{2+}$ ATPase flux is (37)

$$
J_{\text {pump }}=v_{\text {pump }} \frac{\left(\mathrm{c}_{\mathrm{myo}} / K_{f \mathrm{~s}}\right)^{\eta_{f \mathrm{~s}}}-\left(\mathrm{c}_{\mathrm{nsr}} / K_{\mathrm{rs}}\right)^{\eta_{\mathrm{rs}}}}{1+\left(\mathrm{c}_{\mathrm{myo}} / K_{f \mathrm{~s}}\right)^{\eta_{f \mathrm{~s}}}+\left(\mathrm{c}_{\mathrm{nsr}} / K_{\mathrm{rs}}\right)^{\eta_{\mathrm{rs}}}} .
$$




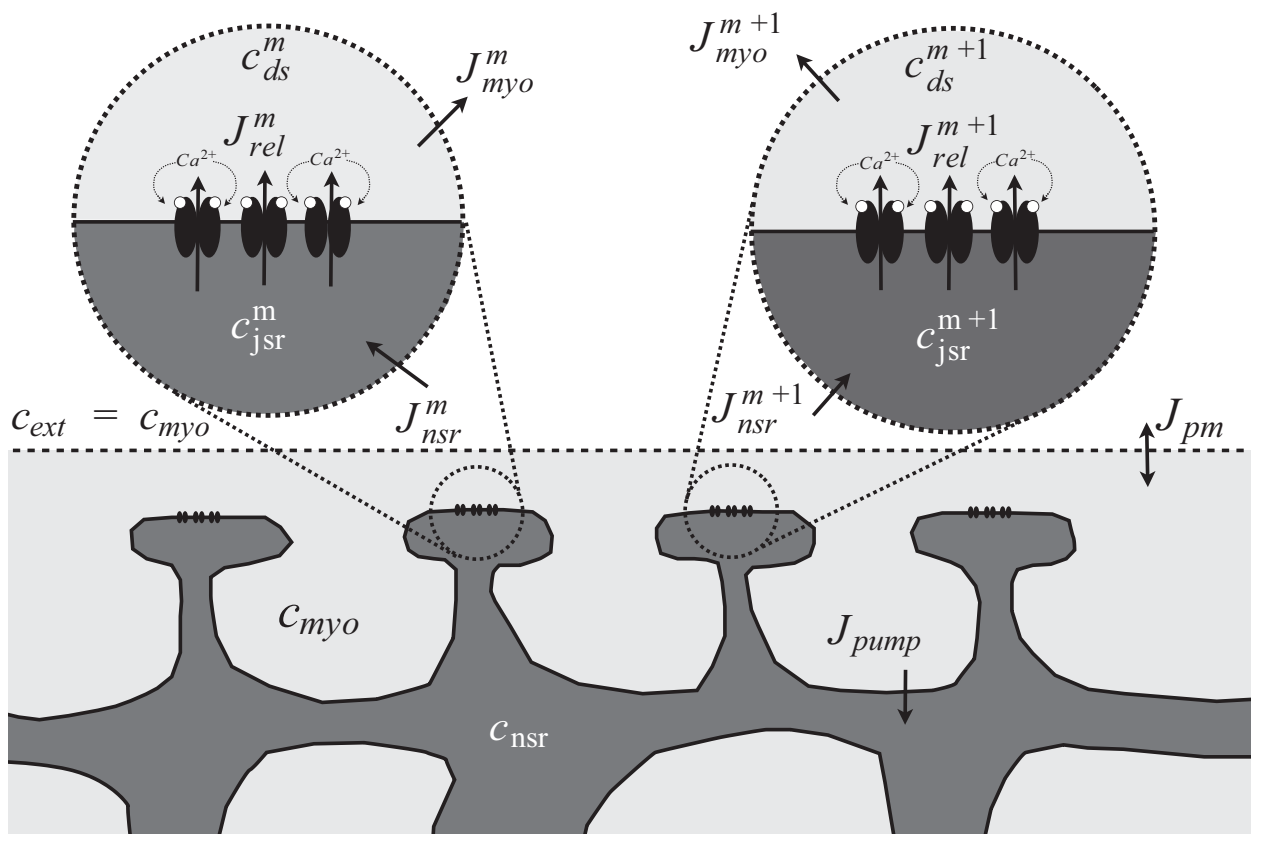

Fig. 2. Diagram of compartments and $\mathrm{Ca}^{2+}$ fluxes in the local/global whole cell model. The model includes $\left[\mathrm{Ca}^{2+}\right]$ in 2 bulk compartments: network sarcoplasmic reticulum (SR) ( $\mathrm{c}_{\mathrm{nsr}}$ ) and myoplasm ( $\left.\mathrm{c}_{\mathrm{myo}}\right) \cdot M \mathrm{Ca}^{2+}$ release units (CaRUs) are coupled to these bulk compartments; each includes a dyadic subspace $\left(\mathrm{c}_{\mathrm{ds}}^{m}\right)$ and junctional SR $\left(\mathrm{c}_{\mathrm{jsr}}^{m}\right)$. The bulk myoplasmic $\left[\mathrm{Ca}^{2+}\right]$ is a model parameter, as this quantity is clamped in permeabilized ventricular myocytes. Fluxes include passive exchange between network and junctional SR $\left(J_{\mathrm{nsr}}^{m}\right)$ and between dyadic subspace and myoplasm $\left(J_{\text {myo }}^{m}\right)$; release fluxes between junctional SR and dyadic subspace $\left(J_{\text {rel }}^{m}\right)$; SR uptake from myoplasm to network SR via SR $\mathrm{Ca}^{2+}$-ATPase (SERCA) $\left(J_{\text {pump }}\right)$; and (for intact cells) plasma membrane fluxes $\left(J_{\mathrm{pm}}\right)$.

The aggregate fluxes $J_{\text {myo }}^{\mathrm{T}}=\sum_{m=1}^{M} J_{\text {myo }}^{m}$ and $J_{\text {nsr }}^{\mathrm{T}}=\sum_{m}^{M}={ }_{1} J_{\mathrm{nsr}}^{m}$ in Eqs. 16 and 17 account for the stochastic dynamics of $\mathrm{Ca}^{2+}$ release, where $J_{\text {myo }}^{\mathrm{T}}=v_{\mathrm{myo}}\left(\mathrm{c}_{\mathrm{ds}}^{m}-\mathrm{c}_{\text {myo }}\right)$ with $v_{\text {myo }}=v_{\text {myo }}^{\mathrm{T}} / M$ is the flux from the $m$ th dyadic subspace into the bulk myoplasm and $J_{\mathrm{nsr}}^{m}=v_{\mathrm{nsr}}\left(\mathrm{c}_{\mathrm{nsr}}-\mathrm{c}_{\mathrm{jsr}}^{m}\right)$ where $v_{\mathrm{nsr}}=v_{\mathrm{nsr}}^{\mathrm{T}} / M$ is the flux from the network SR to the $m$ th junctional SR $(m=1,2, \ldots, M)$. See Table 1 for parameters.

Each CaRU in the whole cell model is a collection of $N$ RyRs with open fraction $f_{\mathrm{O}}^{m}$ and associated dyadic subspace $\left(\mathrm{c}_{\mathrm{ds}}^{m}\right)$ and junctional $\mathrm{SR}\left(\mathrm{c}_{\mathrm{jsr}}^{m}\right) \mathrm{Ca}^{2+}$ concentrations:

$$
\begin{gathered}
\frac{\mathrm{dc}_{\mathrm{ds}}^{m}}{\mathrm{~d} t}=\frac{1}{\lambda_{\mathrm{ds}}}\left(J_{\mathrm{rel}}^{m}-J_{\mathrm{myo}}^{m}\right) \\
\frac{\mathrm{d} f_{\mathrm{O}}^{m}}{\mathrm{~d} t}=k^{+}\left(\mathrm{c}_{\mathrm{ds}}^{m}\right)^{\eta}\left(1-f_{\mathrm{O}}^{m}\right)-k^{-} f_{\mathrm{O}}^{m}+\xi^{m}(t)
\end{gathered}
$$

$$
\frac{\mathrm{dc}_{\mathrm{jsr}}^{m}}{\mathrm{~d} t}=\frac{1}{\lambda_{\mathrm{jsr}}}\left(-J_{\mathrm{rel}}^{m}+J_{\mathrm{nsr}}^{m}\right) .
$$

In these equations, $\lambda_{\mathrm{ds}}$ and $\lambda_{\mathrm{jsr}}$ are effective volume ratios, that is, $\lambda_{\mathrm{ds}}=\left(V_{\mathrm{ds}} / \beta_{\mathrm{ds}}\right) /\left(V_{\mathrm{myo}} / \beta_{\mathrm{myo}}\right)$, where $V_{\mathrm{ds}}=V_{\mathrm{ds}}^{\mathrm{T}} / M$ and $V_{\mathrm{ds}}^{\mathrm{T}}$ is the aggregate volume of the diadic subspaces (similarly for $\lambda_{\text {jsr }}$ ). $J_{\text {rel }}^{m}$ is the release flux though the $m$ th RyR cluster given by $J_{\text {rel }}^{m}=v_{\text {rel }} f_{\mathrm{O}}^{m}\left(\mathrm{c}_{\mathrm{jsr}}^{m}-\right.$ $\mathrm{c}_{\mathrm{ds}}^{m}$ ) for $m=1,2, \ldots, M$ and $v_{\mathrm{rel}}^{\mathrm{T}} / M$. The random functions of time $\xi^{m}(t)$ are indepenent Gaussian white noise terms with zero mean, $\left\langle\xi^{m}(t)\right\rangle=0$ for all $m$, and the two-time covariances are

$$
\left\langle\xi^{m}(t) \xi^{m^{\prime}}\left(t^{\prime}\right)\right\rangle= \begin{cases}0 & \text { for } m \neq m^{\prime} \\ \gamma\left(f_{O}^{m}\right) \delta\left(t-t^{\prime}\right) & \text { for } m=m^{\prime}\end{cases}
$$

\begin{tabular}{|c|c|c|c|}
\hline Parameter & Definition & Units & Value \\
\hline$\Lambda_{\text {nsr }}$ & Effective volume ratio of network SR and myoplasm & - & 1.46 \\
\hline$v_{\text {myo }}^{T}=M v_{\text {myo }}$ & Rate of myoplasmic domain collapse & $\mathrm{s}^{-1}$ & 31.25 \\
\hline$v_{\mathrm{rel}}^{\mathrm{TST}}=M v_{\mathrm{rel}}$ & Maximum release rate via RyRs & $\mathrm{s}^{-1}$ & 1.56 \\
\hline$v_{\text {pump }}$ & Maximum pump rate via SERCA & $\mu \mathrm{M} / \mathrm{s}$ & 161.25 \\
\hline$K_{\mathrm{fs}}, K_{\mathrm{rs}}$ & Forward and reverse half-saturation constant & $\mu \mathrm{M}$ & $0.17,1702$ \\
\hline$k^{-}$ & Disassociation rate constant for $\mathrm{Ca}^{2+}$ unbinding & $\mathrm{s}^{-1}$ & 50 \\
\hline$\eta$ & Cooperativity of $\mathrm{Ca}^{2+}$ binding to RyRs & - & 2 \\
\hline$M$ & Number of CaRUs in Langevin simulations & - & 200 \\
\hline
\end{tabular}

where

Table 1. Parameters for the local/global whole cell model of calcium homeostasis in permeabilized ventricular myocytes

Myoplasmic $\mathrm{Ca}^{2+}$ concentration ( $\mathrm{c}_{\text {myo }}$ ) is under experimental control in permeabilized myocytes, and thus it is a parameter of the whole cell model. The effective volume ratio that accounts for $\mathrm{Ca}^{2+}$ buffering in $E q .17$ is given by $\Lambda_{\text {nsr }}=\left(V_{\text {nsr }} / \beta_{\text {nsr }}\right) /\left(V_{\text {myo }} / \beta_{\text {myo }}\right)$, where $V_{\text {nsr }}$ and $V_{\text {myo }}$ are the volume of network sarcoplasmic reticulum (SR) and myoplasm, respectively, and $\beta_{\mathrm{nsr}}$ and $\beta_{\mathrm{myo}}$ are the buffer factors of network SR and myoplasm, respectively. The rate constants $v_{\text {myo }}^{\mathrm{T}}, v_{\text {nsr }}^{\mathrm{T}}$, and $v_{\text {rel }}^{\mathrm{T}}$ scale the fluxes between domains and bulk, given by integrals over the density function $\rho(f, t): J_{\text {myo }}^{\mathrm{T}}=\int v_{\text {myo }}^{\mathrm{T}}\left(\bar{c}_{\mathrm{ds}}-c_{\text {myo }}\right) \rho(f, t) \mathrm{d} f$, $J_{\text {rel }}^{\mathrm{T}}=\int v_{\text {rel }}^{\mathrm{T}} f\left(\bar{c}_{\mathrm{jsr}}-\bar{c}_{\mathrm{ds}}\right) \rho(f, t) \mathrm{d} f$, and $J_{\text {nsr }}^{\mathrm{T}}=\int v_{\mathrm{nsr}}^{\mathrm{T}}\left(c_{\mathrm{nsr}}-\bar{c}_{\mathrm{jsr}}\right) \rho(f, t) \mathrm{d} f$, where $\bar{c}_{\mathrm{ds}}(f)$ and $\bar{c}_{\mathrm{jsr}}(f)$ are given by Eqs. 32 and 33 . The flux via SR Ca ${ }^{2+}-\mathrm{ATPase}(\mathrm{SERCA})$ is governed by $v_{\mathrm{pump}}, K_{\mathrm{fs}}, K_{\mathrm{rs}}, \eta_{\mathrm{fs}}$, and $\eta_{\mathrm{rs}}(E q .18)$. $\mathrm{Ca}^{2+}$ activation and dissociation of the 2-state ryanodine receptor (RyR) channel model are governed by $k^{+}$, $k^{-}$, and $\eta(E q .1)$. The number of $\mathrm{Ca}^{2+}$ release units $(\mathrm{CaRUs} ; M)$ in the Fokker-Planck formulation is large but unspecified. 


$$
\gamma\left(f_{\mathrm{O}}^{m}\right)=\frac{k^{+}\left(\mathrm{c}_{\mathrm{ds}}^{m}\right)^{\eta}\left(1-f_{\mathrm{O}}^{m}\right)+k^{-} f_{\mathrm{O}}^{m}}{N} .
$$

Note that the dyadic subspaces only influence each other through the bulk concentrations $\mathrm{c}_{\mathrm{myo}}$ and $\mathrm{c}_{\mathrm{nsr}}$. Below we refer to Eqs. 16-23 as the "full local/global whole cell model."

Reduced local/global whole cell model. The Langevin description of each CaRU (Eqs. 19-21) in the full local/global model may be simplified by assuming that the dyadic subspace and junctional SR rapidly equilibrate with the bulk myoplasmic and network $\mathrm{SR}\left[\mathrm{Ca}^{2+}\right]$, that is, $J_{\text {rel }}^{m}=J_{\text {myo }}^{m}$ and $J_{\text {rel }}^{m}=J_{\text {nsr }}^{m}$. These balanced fluxes relate the $2 M$ domain $\mathrm{Ca}^{2+}$ concentrations, $\mathrm{c}_{\mathrm{ds}}^{m}$ and $\mathrm{c}_{\mathrm{jsr}}^{m}$, to the bulk concentrations, $\mathrm{c}_{\mathrm{myo}}$ and $\mathrm{c}_{\mathrm{nsr}}$, and the fraction of open channels $f_{\mathrm{O}}^{m}$ in the $m$ th CaRU as follows (22),

$$
\begin{aligned}
& \overline{\mathrm{c}}_{\mathrm{ds}}^{m}=\left(1-\chi_{\text {myo }}^{m}\right) \mathrm{c}_{\mathrm{myo}}+\chi_{\text {myo }}^{m} \mathrm{c}_{\mathrm{nsr}} \\
& \overline{\mathrm{c}}_{\mathrm{jsr}}^{m}=\chi_{\text {nsr }}^{\mathrm{m}} \mathrm{c}_{\mathrm{myo}}+\left(1-\chi_{\mathrm{nsr}}^{\mathrm{m}}\right) \mathrm{c}_{\mathrm{nsr}},
\end{aligned}
$$

where $\chi_{\text {myo }}^{m}=\tilde{v}_{\text {nsr }}^{m} /\left(v_{\text {myo }}+\tilde{v}_{\text {nsr }}^{m}\right)$ and $\chi_{\text {nsr }}^{m}=\tilde{v}_{\text {myo }}^{m} /\left(v_{\text {nsr }}+\tilde{v}_{\text {myo }}^{m}\right)$, $\tilde{v}_{\mathrm{nsr}}^{m}=\tilde{v}_{\mathrm{rel}}^{m} v_{\mathrm{nsr}} /\left(\tilde{v}_{\mathrm{rel}}^{m}+v_{\mathrm{nsr}}\right), \tilde{v}_{\text {myo }}^{m}=\tilde{v}_{\mathrm{rel}}^{m} v_{\mathrm{myo}} /\left(\tilde{v}_{\mathrm{rel}}^{m}+v_{\text {myo }}\right), \tilde{v}_{\mathrm{rel}}^{m}=v_{\text {rel }} f_{\mathrm{O}}^{m}$. Equations 24 and 25 eliminate $2 M$ of the $3 M$ ODEs representing the population of $M$ CaRUs, with the remaining ODEs,

$$
\frac{\mathrm{d} f_{\mathrm{O}}^{m}}{\mathrm{~d} t}=k^{+}\left(\overline{\mathrm{c}}_{\mathrm{ds}}^{m}\right)^{\eta}\left(1-f_{\mathrm{O}}^{m}\right)-k^{-} f_{\mathrm{O}}^{m}+\xi^{m}(t),
$$

dependent on the rapidly equilibrated dyadic subspace concentration $\overline{\mathrm{c}}_{m}^{\mathrm{ds}}$ that is an algebraic function of $f_{\mathrm{O}}^{m}, \mathrm{c}_{\mathrm{myo}}$, and $\mathrm{c}_{\mathrm{nsr}}$. Realizations of this "reduced local/global whole cell model" are obtained by numerically integrating Eqs. 16, 17, and 26.

Fokker-Planck local/global whole cell model. The full and reduced local/global whole cell models presented above include heterogeneous local $\mathrm{Ca}^{2+}$ signaling and stochastic $\mathrm{Ca}^{2+}$ release. Unfortunately, a physiologically realistic ventricular myocyte simulation would involve $M \approx 20,000$ CaRUs (6). Rather than perform Monte Carlo simulations with a lesser, unphysiological value for $M$ that is computationally feasible, we recognize that a Fokker-Planck equation similar to $E q .9$ is the master equation for a CaRU and its associated domains. Because the $M$ CaRUs in the whole cell model are identical and independent except for fluxes to and from the bulk myoplasm and network SR, we replace the $M$ SDEs representing these CaRUs (Eq. 26 ) with this Fokker-Planck equation (a good approximation for large $M$ that is exact as $M \rightarrow \infty)$. In this way, we obtain the "Fokker-Planck local/global whole cell model."

In the study of $\mathrm{Ca}^{2+}$ homeostasis in permeabilized ventricular myocytes presented below, the governing equations are Eqs. 9, 16, and 17 , with the fluxes $J_{\text {myo }}^{\mathrm{T}}$ and $J_{\text {nsr }}^{\mathrm{T}}$ redefined as functions of the probability distribution function for $f_{\mathrm{O}}$ in a randomly sampled CaRU. In permeabilized myocytes, the bulk myoplasmic $\left[\mathrm{Ca}^{2+}\right]$ is clamped ( $k_{\mathrm{pm}}$ is large) and $\mathrm{c}_{\mathrm{myo}} \approx \mathrm{c}_{\mathrm{ext}}$ is no longer a variable but a parameter. Consequently, Eq. 16 is superfluous and the governing equations for the Fokker-Planck equation description of the local/global model of permeabilized ventricular myocytes are therefore given by

$$
\begin{gathered}
\frac{\mathrm{d} c_{\mathrm{nsr}}}{\mathrm{d} t}=\frac{1}{\Lambda_{\mathrm{nsr}}}\left(-J_{\mathrm{nsr}}^{\mathrm{T}}+J_{\text {pump }}\right) \\
\frac{\partial \rho}{\partial t}=-\frac{\partial}{\partial f}[\alpha \rho]+\frac{1}{2} \frac{\partial^{2}}{\partial f^{2}}[\gamma \rho]
\end{gathered}
$$

where

$$
J_{\mathrm{nsr}}^{\mathrm{T}}(t)=v_{\mathrm{nsr}}^{\mathrm{T}} \int\left(\mathrm{c}_{\mathrm{nsr}}-\overline{\mathrm{c}}_{\mathrm{jsr}}\right) \rho(f, t) \mathrm{d} f .
$$

In Eq. 28, $\alpha(f)$ and $\gamma(f)$ are given by Eqs. 10 and 11, with

$$
\begin{gathered}
v^{+}=k^{+} \bar{c}_{\mathrm{ds}}^{\eta}(1-f), \\
v^{-}=k^{-} f .
\end{gathered}
$$

The equilibrated domain concentrations are given by

$$
\begin{gathered}
\overline{\mathrm{c}}_{\mathrm{ds}}=\left[\left(1-\chi_{\mathrm{myo}}\right) \mathrm{c}_{\mathrm{myo}}+\chi_{\mathrm{myo}} c_{\mathrm{nsr}}\right] \\
\overline{\mathrm{c}}_{\mathrm{jsr}}=\left[\chi_{\mathrm{nsr}} c_{\mathrm{myo}}+\left(1-\chi_{\mathrm{nsr}}\right) c_{\mathrm{nsr}}\right]
\end{gathered}
$$

where $\chi_{\text {myo }}$ and $\chi_{\mathrm{nsr}}$ are the following functions of $f$,

$$
\begin{aligned}
& \chi_{\text {myo }}=\tilde{v}_{\text {nsr }}^{\mathrm{T}} /\left(v_{\text {myo }}^{\mathrm{T}}+\widetilde{v}_{\text {nsr }}^{\mathrm{T}}\right) \\
& \chi_{\mathrm{nsr}}=\widetilde{v}_{\text {myo }}^{\mathrm{T}} /\left(v_{\mathrm{nsr}}^{\mathrm{T}}+\widetilde{v}_{\text {myo }}^{\mathrm{T}}\right)
\end{aligned}
$$

where

$$
\begin{gathered}
\tilde{v}_{\text {nsr }}^{\mathrm{T}}=\tilde{v}_{\text {rel }}^{\mathrm{T}} v_{\text {nsr }}^{\mathrm{T}} /\left(\tilde{v}_{\text {rel }}^{\mathrm{T}}+v_{\mathrm{nsr}}^{\mathrm{T}}\right) \\
\tilde{v}_{\text {myo }}^{\mathrm{T}}=\tilde{v}_{\text {rel }}^{\mathrm{T}} v_{\text {myo }}^{\mathrm{T}} /\left(\tilde{v}_{\text {rel }}^{\mathrm{T}}+v_{\text {myo }}^{\mathrm{T}}\right)
\end{gathered}
$$

and $\widetilde{v}_{\text {rel }}^{\mathrm{T}}=v_{\text {rel }}^{\mathrm{T}}$ (cf. Eqs. 24 and 25). In the local/global whole cell model calculations presented below, the Fokker-Planck equation was numerically integrated using a total variation diminishing scheme (37), with boundary conditions as described in APPENDIX C.

\section{RESULTS}

Calcium homeostasis in the local/global whole cell model. We use the Fokker-Planck version of the reduced local/global model (Eqs. 27-33) to investigate $\mathrm{Ca}^{2+}$ homeostasis in permeabilized ventricular myocytes, in particular, the influence of $\mathrm{c}_{\text {myo }}$ on $\mathrm{SR} \mathrm{Ca}^{2+}$ load and release. The relationship between $\mathrm{c}_{\text {myo }}$ and $\mathrm{Ca}^{2+}$ homeostasis is complex, as $\mathrm{c}_{\text {myo }}$ can promote elevated $c_{n s r}$ through increased SERCA uptake. On the other hand, a sufficiently elevated $\mathrm{c}_{\mathrm{nsr}}$ also promotes $\mathrm{Ca}^{2+}$ sparks that may deplete the network SR (i.e., decrease $\mathrm{c}_{\mathrm{nsr}}$ ).

With the use of an intermediate value for the myoplasmic $\left[\mathrm{Ca}^{2+}\right]\left(\mathrm{c}_{\text {myo }}=0.18 \mu \mathrm{M}\right)$ in the permeabilized ventricular myocyte model, Fig. $3 A$ shows the bimodal steady-state probability density function for the fraction of open channels, $\rho_{\mathrm{ss}}(f)$, calculated via the Fokker-Planck version of the whole cell model (solid line). This bimodal density reflects the dynamics of CaRUs composed of RyRs that are usually closed but occasionally open in a concerted fashion. For comparison, Fig. $3 A$ also shows a (nearly identical) estimate of the steady-state density function obtained from a whole cell model with the corresponding Langevin description of $M=200$ release sites (dashed curve). Figure $3 B$ compares the stationary distribution for a whole cell model that uses a Markov chain description of release sites (white bars) and the corresponding distribution calculated via the Fokker-Planck version of the model (appropriately discretized). The two histograms are qualitatively identical and in strong qualitative agreement (the Markov chain simulation is slightly shifted to larger $f_{\mathrm{O}}$ ), validating the minimal Fokker-Planck formulation of the local/global whole cell model (Eqs. 27-37).

With the use of the Fokker-Planck-based whole cell model, Fig. $3 C$ shows the monotone increasing relationship between the fraction of open channels and stochastic $\mathrm{Ca}^{2+}$ release rate, given by $v_{\text {rel }} f\left(\overline{\mathrm{c}}_{\mathrm{jsr}}-\overline{\mathrm{c}}_{\mathrm{ds}}\right)$ where $\overline{\mathrm{c}}_{\mathrm{jsr}}$ and $\overline{\mathrm{c}}_{\mathrm{ds}}$ are functions of $f$ (Eqs. 32 and 33). Figure $3 D$ shows the steady-state release flux density, given by $v_{\mathrm{rel}} f\left(\overline{\mathrm{c}}_{\mathrm{jsr}}-\overline{\mathrm{c}}_{\mathrm{ds}}\right) \rho_{\mathrm{ss}}$, that is, the product of the curves in Fig. 3, $A$ and $C$. Note that the steady-state release flux density is also a bimodal function of $f$, with the first and second modes corresponding to nonspark-mediated (light gray area, $f<0.1$ ) and spark-mediated stochastic $\mathrm{Ca}^{2+}$ release (dark gray area, $f \geq 0.1$ ), respectively.

Figure 4 shows steady-state values for total release flux $\left(J_{\text {rel }}^{\mathrm{T}}\right)$, network SR $\left[\mathrm{Ca}^{2+}\right]\left(\mathrm{c}_{\mathrm{nsr}}\right)$, and the spark Score as a 
A
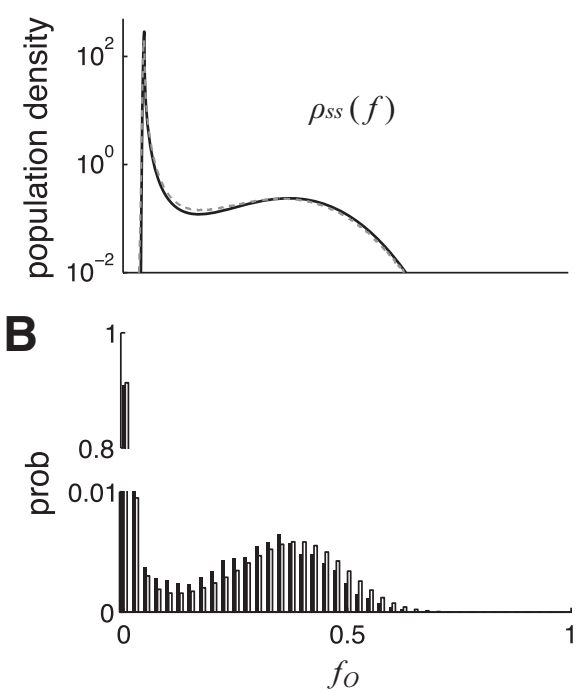
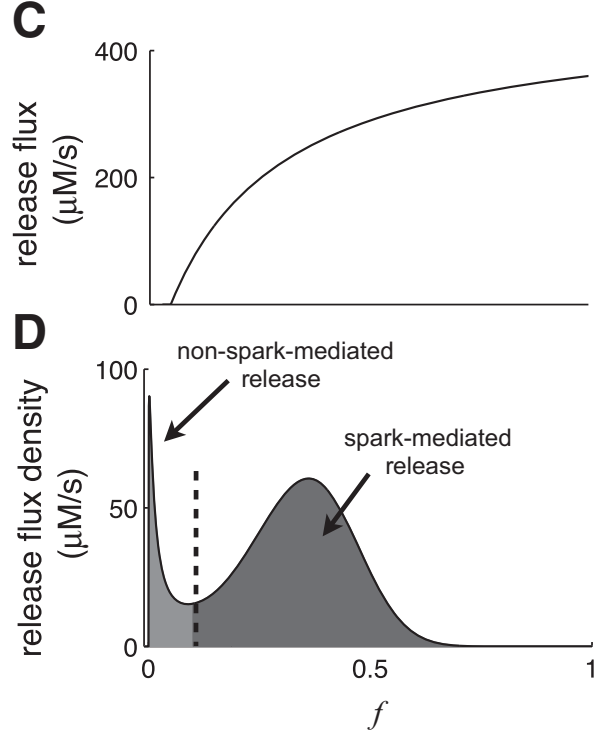

Fig. 3. Spark- and nonspark-mediated release from the population of CaRUs represented in the local/global whole cell model. A: steady-state density function $\rho_{\mathrm{ss}}(f)$ of the Fokker-Planck-based whole cell model (solid line) and the corresponding density in the Langevin model (dashed line, $M=200$ release sites). $B$ : binned (discretized) steady-state density of the Fokker-Planck model (black histogram) and distribution of the number of open channels in the Markov chain-based whole cell model (white histogram, average of $M=200$ release sites). $C$ and $D$ : RyR release rate function $(C)$ given by $v_{\text {rel }} f\left(\overline{\mathrm{c}}_{\mathrm{jsr}}-\overline{\mathrm{c}}_{\mathrm{ds}}\right)$ and the steady-state release flux density $(D)$ given by $v_{\text {rel }} f\left(\bar{c}_{\mathrm{jsr}}-\overline{\mathrm{c}}_{\mathrm{ds}}\right) \rho_{\mathrm{ss}}$, plotted as functions of the fraction of open channels $(f)$. Parameters: $\mathrm{c}_{\mathrm{myo}}=$ $0.18 \mu \mathrm{M}, N=40$ RyRs per CaRU. In this figure and those follow, see Table 1 for other parameters. function of $\mathrm{c}_{\mathrm{myo}}$, obtained from simulation of the local/global model using the Langevin (+ symbols) and Fokker-Planck (solid lines) descriptions of the CaRU population. The spark Score is the index of dispersion of the fraction of open channels $\left(f_{\mathrm{O}}\right)$,

$$
\text { Score }=\frac{\operatorname{Var}\left[f_{\mathrm{O}}\right]}{\mathrm{E}\left[f_{\mathrm{O}}\right]},
$$

where $\mathrm{E}\left[f_{\mathrm{O}}\right]=\int f \rho_{\mathrm{ss}} \mathrm{d} f, \operatorname{Var}\left[f_{\mathrm{O}}\right]=\int\left(f-\mathrm{E}\left[f_{\mathrm{O}}\right]\right)^{2} \rho_{\mathrm{ss}} \mathrm{d} f$, and $\rho_{\mathrm{ss}}(f)$ is the steady-state probability density of open channels. The spark Score takes values between 0 and 1, and a Score greater than $\sim 0.25$ indicates the presence of robust $\mathrm{Ca}^{2+}$ sparks (21). Over a wide range of $\mathrm{c}_{\text {myo }}$ values, there is agreement among $J_{\text {rel }}^{\mathrm{T}}, \mathrm{c}_{\mathrm{nsr}}$, and the spark Score calculated using the Langevin and Fokker-Planck approaches, validating the use of the FokkerPlanck version of the model and our implementation of both methods. Note that $J_{\text {rel }}^{\mathrm{T}}$ is a monotone increasing function of $\mathrm{c}_{\text {myo }}$ (Fig. $4 A$ ), while $\mathrm{c}_{\mathrm{nsr}}$ is biphasic, increasing for $\mathrm{c}_{\text {myo }}<0.2$ $\mu \mathrm{M}$ and decreasing for $\mathrm{c}_{\text {myo }}>0.2 \mu \mathrm{M}$ (Fig. $4 B$ ). The spark Score shows similar biphasic dependence on $\mathrm{c}_{\mathrm{myo}}$ (Fig. $4 C$ ).

The biphasic dependence of $\mathrm{c}_{\mathrm{nsr}}$ and the spark Score on $\mathrm{c}_{\mathrm{myo}}$ can be understood by considering the representative stochastic trajectories for the fraction of open channels in a randomly sampled CaRU in the Langevin model (Fig. 4A) or, alternatively, the steady-state population density function $\rho_{\mathrm{ss}}(f)$ in the Fokker-Planck model (Fig. $4 C$ ). For a low myoplasmic $\left[\mathrm{Ca}^{2+}\right]$ $\left(\mathrm{c}_{\text {myo }}=0.1 \mu \mathrm{M}\right), \rho_{\mathrm{ss}}(f)$ is located near $f=0$, consistent with few channel openings (Fig. $4, A$ and $C$, insets). As $\mathrm{c}_{\text {myo }}$ increases to an intermediate value of $0.2 \mu \mathrm{M}$, increased SERCA uptake elevates $c_{n s r}$ and $\rho_{\mathrm{ss}}(f)$ is distinctly bimodal, consistent with robust sparks and the observed increase in $J_{\text {rel }}^{\mathrm{T}}$ and Score. However, a further increase in myoplasmic $\left[\mathrm{Ca}^{2+}\right]$ $\left(\mathrm{c}_{\text {myo }}=0.6 \mu \mathrm{M}\right)$ promotes tonic activation of CaRUs (as opposed to sparks, for a decreasing Score). The resulting increase in release flux $\left(J_{\text {rel }}^{\mathrm{T}}\right)$ depletes the network SR $\left[\mathrm{Ca}^{2+}\right]$ (lower values of $\mathrm{c}_{\mathrm{nsr}}$ ) and eliminates robust sparks. APPENDIX D
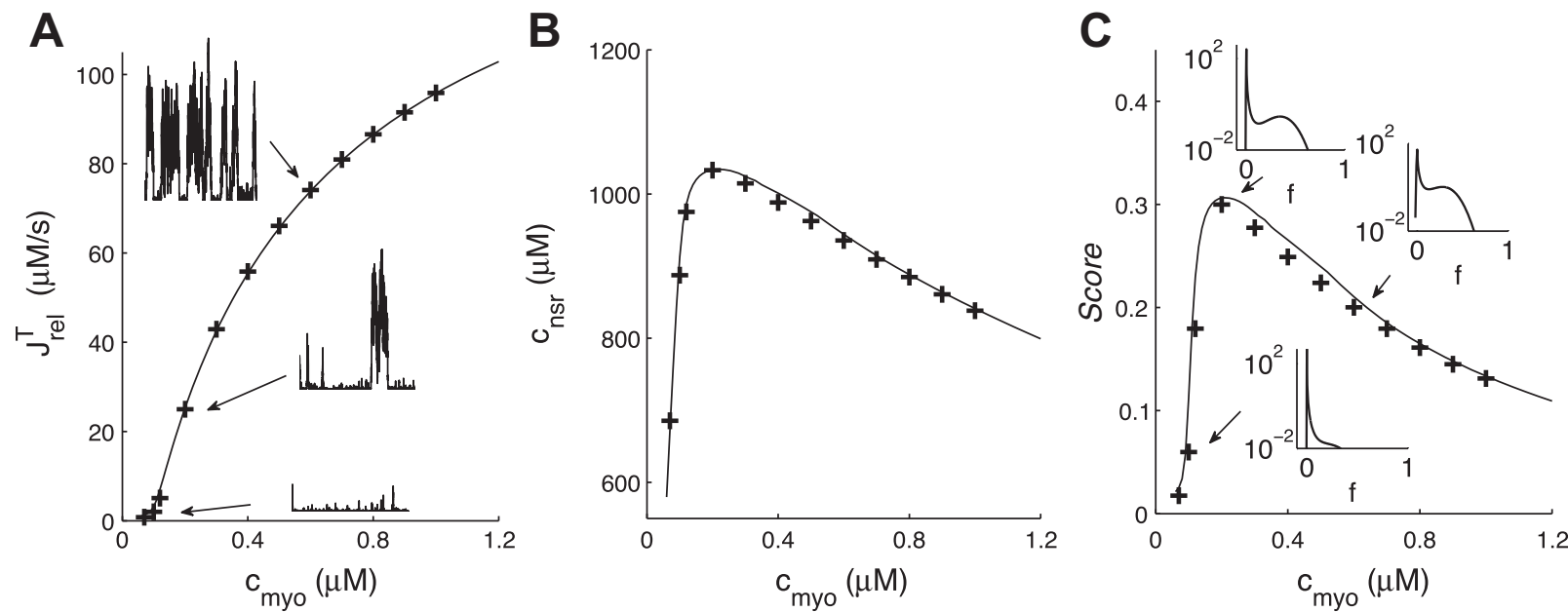

Fig. 4. $A-C$ : steady-state values for total RyR release flux $J_{\text {rel }}^{\mathrm{T}}(A)$, network SR $\left[\mathrm{Ca}^{2+}\right]\left(\mathrm{c}_{\mathrm{nsr}} ; B\right)$, and spark Score $(C)$ as a function of myoplasmic $\left[\mathrm{Ca}^{2+}\right]\left(\mathrm{c}_{\mathrm{myo}}\right)$ as calculated using the Fokker-Planck local/global whole cell model (solid black lines). The + symbols indicate the average across 10 simulations, each $20 \mathrm{~s}$ in duration, of the Langevin version of the model (with $M=200$ CaRUs). Insets: sample trajectories from the Langevin model $(A)$ and the steady-state population density function $\rho_{\mathrm{ss}}(f)$ from the Fokker-Planck model $(C)$, respectively, for $\mathrm{c}_{\mathrm{myo}}=0.1,0.2$, and $0.6 \mu \mathrm{M}$. 


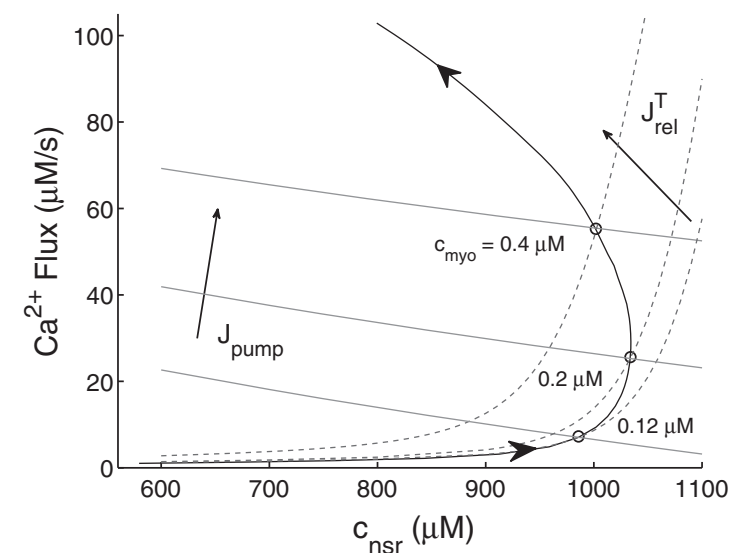

Fig. 5. Balance between SR $\mathrm{Ca}^{2+}$ release and uptake at steady state. The SR $\mathrm{Ca}^{2+}$ release flux $J_{\text {rel }}^{\mathrm{T}}$ (black dashed lines) and SERCA uptake flux $J_{\text {pump }}$ (solid gray lines) are shown as a function of a fixed (clamped) network SR $\left[\mathrm{Ca}^{2+}\right]$ (as though $\mathrm{c}_{\mathrm{nsr}}$ were a parameter) for different values of myoplasmic $\left[\mathrm{Ca}^{2+}\right]$ $\left(\mathrm{c}_{\mathrm{myo}}\right)$. Steady-state release fluxes (solid black lines) are shown as a function of clamped $\mathrm{c}_{\mathrm{nsr}}$ for increasing values of $\mathrm{c}_{\mathrm{myo}}$ from 0.06 to $1.2 \mu \mathrm{M}$ (arrows). Each intersection of these curves ( 3 open circles) indicates a steady-state release flux $\left(J_{\text {rel }}^{\mathrm{T}}\right)$ and corresponding unclamped SR $\mathrm{Ca}^{2+}$ load $\left(\mathrm{c}_{\mathrm{nsr}}\right)$ solving Eqs. 27-37 for a particular $\mathrm{c}_{\mathrm{myo}}$.

provides more details regarding the influences of $\mathrm{c}_{\mathrm{myo}}$ on steady-state spark statistics.

In Fig. $5, J_{\text {rel }}^{\mathrm{t}}$ (black dashed lines) and $J_{\text {pump }}$ (solid gray lines) are shown as a function of $\mathrm{c}_{\mathrm{nsr}}$ for three values of $\mathrm{c}_{\mathrm{myo}} . J_{\mathrm{rel}}^{\mathrm{T}}$ is a monotone increasing function of $\mathrm{c}_{\mathrm{nsr}}$; the increasing slope at high $\mathrm{c}_{\mathrm{nsr}}$ levels is due to spark-mediated $\mathrm{Ca}^{2+}$ release. $J_{\text {pump }}$ decreases approximately linearly with $\mathrm{c}_{\mathrm{nsr}}$, and both $J_{\text {rel }}^{\mathrm{T}}$ and $J_{\text {pump }}$ increase for increasing $\mathrm{c}_{\text {myo }}$. The intersection of the $J_{\text {rel }}^{\mathrm{T}}$ and $J_{\text {pump }}$ curves (open circles) indicate the steady-state total release flux and $\mathrm{SR} \mathrm{Ca}^{2+}$ load $\left(\mathrm{c}_{\mathrm{nsr}}\right)$ for a given value of $\mathrm{c}_{\mathrm{myo}}$ (solid black line, arrow indicates increasing $\mathrm{c}_{\mathrm{myo}}$ ). For a given $\mathrm{c}_{\mathrm{nsr}}$, two distinct steady-states are possible, one with low $\mathrm{c}_{\mathrm{myo}}$ and $J_{\text {rel }}^{\mathrm{T}}$ (primarily nonspark-mediated release) and another with high $\mathrm{c}_{\mathrm{myo}}$ and $J_{\text {rel }}^{\mathrm{T}}$ (primarily spark-mediated release). The next section further explores the dependence of spark- and nonspark-mediated release on $\mathrm{c}_{\mathrm{myo}}$.

Spark- and nonspark-mediated SR $\mathrm{Ca}^{2+}$ release. In a recent experimental study, Bovo et al. (3) demonstrated that myoplasmic $\mathrm{Ca}^{2+}$ levels augment both spark-mediated $\mathrm{SR} \mathrm{Ca}^{2+}$ release and nonspark-mediated $\mathrm{SR} \mathrm{Ca}^{2+}$ release in ventricular myocytes (3). While controlling myoplasmic $\left[\mathrm{Ca}^{2+}\right]\left(\mathrm{c}_{\mathrm{myo}}\right)$ by permeabilization of the cell plasma membrane, the time course of network SR $\left[\mathrm{Ca}^{2+}\right]\left(\mathrm{c}_{\mathrm{nsr}}\right)$ depletion was measured following application of the SERCA inhibitor thapsigargin (cf. Ref. 3, Fig. $1 A$ ). Assuming negligible SERCA activity (i.e., $J_{\text {pump }}=$ 0 ), the rate of change of $c_{n s r}$ was used as a measure of the SR $\mathrm{Ca}^{2+}$ release flux (see Eq. 27), and further analysis was performed to distinguish spark- and nonspark-mediated release as functions of $\mathrm{c}_{\mathrm{myo}}$ and $\mathrm{c}_{\mathrm{nsr}}$. Figure 6 uses a similar protocol (setting $v_{\text {pump }}=0$ ) to elucidate the influence of $\mathrm{c}_{\text {myo }}$ on sparkand nonspark-mediated release. Consistent with Bovo et al. and Fig. $4 B$, Fig. 6 shows that steady-state $c_{n s r}$ increases as $c_{m y o}$ increases from 0.12 to $0.18 \mu \mathrm{M}$ (compare initial values, solid, dashed, and thick solid lines). Consistent with the experiment, increasing $\mathrm{c}_{\mathrm{myo}}$ in this range of concentrations also leads to an increased $\mathrm{Ca}^{2+}$ release rate, as evidenced by faster SR deple- tion upon simulated block of SERCA with thapsigargin (TG in Fig. 6).

Figure 7 shows the total release flux, $J_{\text {rel }}^{\mathrm{T}}$, and the spark- and nonspark-mediated release $\left(J_{\text {rel }}^{\mathrm{S}}\right.$ and $J_{\text {rel }}^{\mathrm{NS}}$ as defined in Fig. $3 C$ ) as a function of $\mathrm{c}_{\mathrm{nsr}}$ during the SR depletion simulation of Fig. 6 for different values of $c_{\text {myo }}$ (cf. Ref. 3, Fig. 3). While $J_{\text {rel }}^{\mathrm{T}}$ increases as a function of both $\mathrm{c}_{\mathrm{nsr}}$ and $\mathrm{c}_{\text {myo }}$ (Fig. $7 A$ ), the contributions of the spark- and nonspark-mediated release $\left(J_{\text {rel }}^{\mathrm{S}}\right.$ and $\left.J_{\text {rel }}^{\mathrm{NS}}\right)$ are highly dependent on $\mathrm{c}_{\text {nsr. }}$. At low network SR $\left[\mathrm{Ca}^{2+}\right]\left(\mathrm{c}_{\mathrm{nsr}}\right)$, the spark-mediated release flux $\left(J_{\text {rel }}^{S}\right)$ is negligible, but it increases exponentially as $\mathrm{c}_{\mathrm{nsr}}$ increases (Fig. $7 B$ ). The nonspark-mediate release $\left(J_{\text {rel }}^{\mathrm{NS}}\right)$ is small for low $\mathrm{c}_{\mathrm{nsr}}$ levels and increases as a linear function of $\mathrm{c}_{\mathrm{nsr}}$ (Fig. $7 \mathrm{C}$ ). When the SR load is clamped at $c_{\text {nsr }}=950 \mu \mathrm{M}$, both $J_{\text {rel }}^{\mathrm{S}}$ and $J_{\text {rel }}^{\mathrm{NS}}$ increase as $\mathrm{c}_{\text {myo }}$ increases (Fig. $7 D$ ). However, the sparkmediated release flux $\left(J_{\text {rel }}^{\mathrm{S}}\right)$ increases to a greater extent than the nonspark-mediated release $\left(J_{\text {rel }}^{\mathrm{NS}}\right)$. Steady-state calculations of $J_{\text {rel }}^{\mathrm{T}}, J_{\text {rel }}^{\mathrm{S}}$, and $J_{\text {rel }}^{\mathrm{NS}}$ closely agree with time-varying simulations (Fig. 7, + symbols). In summary, when the SR is depleted, most SR $\mathrm{Ca}^{2+}$ release occurs via nonspark-mediated release; conversely, when the $\mathrm{SR}$ is replete, most $\mathrm{SR} \mathrm{Ca}^{2+}$ release occurs via $\mathrm{Ca}^{2+}$ sparks, more so as $\mathrm{c}_{\text {myo }}$ increases.

The number of RyRs per CaRU, $N$, can vary over a wide physiological range (11). Figure 8 shows the steady-state values for $J_{\text {rel }}^{\mathrm{T}}, J_{\text {rel }}^{\mathrm{S}}$, and $J_{\text {rel }}^{\mathrm{NS}}$ for different values of $N$. As $N$ increases (scaling $v_{\text {rel }}$ appropriately such that $J_{\text {rel }}^{\mathrm{T}}$ when all $N$ channel are open is unchanged), $J_{\text {rel }}^{\mathrm{T}}$ becomes a steeper function of $\mathrm{c}_{\mathrm{nsr}}$ (Fig. 8A). Interestingly, when the network SR $\left[\mathrm{Ca}^{2+}\right]$ is higher $\left(\mathrm{c}_{\mathrm{nsr}}=1,000 \mu \mathrm{M}\right), J_{\text {rel }}^{\mathrm{T}}$ is larger for large $N$, but when $\mathrm{c}_{\mathrm{nsr}}$ is slightly lower $\left(\mathrm{c}_{\mathrm{nsr}}=950 \mu \mathrm{M}\right), J_{\text {rel }}^{\mathrm{T}}$ is smaller for large $N$ (Fig. $8 A$, arrows). Spark-mediated release $\left(J_{\text {rel }}^{\mathrm{S}}\right)$ varies with $N$ in a manner similar to $J_{\text {rel }}^{\mathrm{T}}$ (Fig. $8 B$ ), while nonspark-mediated release $\left(J_{\text {rel }}^{\mathrm{NS}}\right)$ generally decreases as $N$ increases (Fig. $8 C$ ).

Figure 9 shows how steady-state probability density function, $\rho_{\mathrm{ss}}(f)$, and the release flux density, $v_{\text {rel }}^{\mathrm{T}} f\left(\overline{\mathrm{c}}_{\mathrm{jsr}}-\overline{\mathrm{c}}_{\mathrm{ds}}\right) \rho_{\mathrm{ss}}$, depend on the number of RyRs per release site $(N)$ when the total release rate $v_{\text {rel }}^{\mathrm{T}}$ is fixed (i.e., $M N$ is a constant). For network SR $\left[\mathrm{Ca}^{2+}\right]$ of $\mathrm{c}_{\mathrm{nsr}}=950 \mu \mathrm{M}$ (Fig. 9A), a larger number of channels per CaRU $(N)$ decreases the "diffusion" term (channel gating fluctuations) in Eq. 11 and both spark-

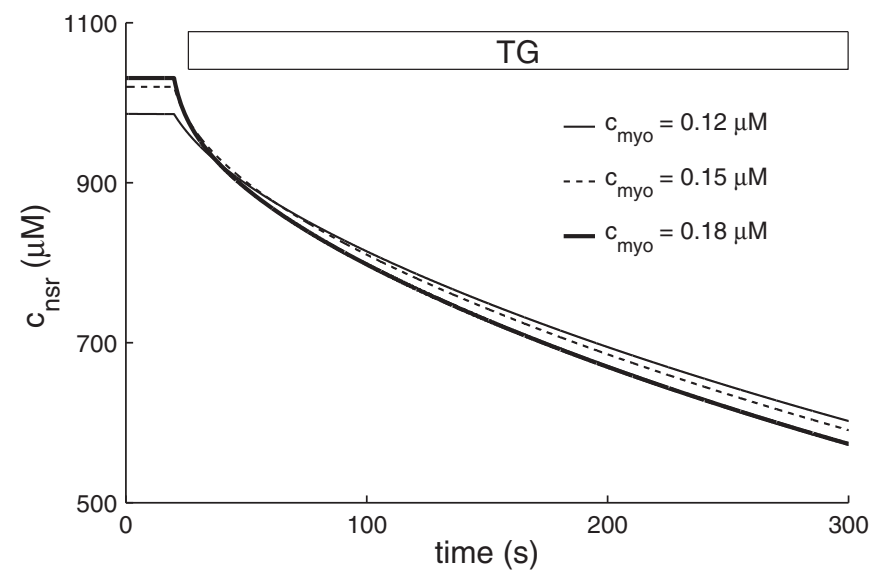

Fig. 6. SR $\mathrm{Ca}^{2+}$ depletion following inhibition of SERCA uptake in permeabilized ventricular myocytes. Network SR $\mathrm{Ca}^{2+}$ concentration, $\mathrm{c}_{\mathrm{nsr}}$, is shown as a function of time for different values of myoplasmic $\mathrm{Ca}^{2+}$ concentration, $\mathrm{c}_{\mathrm{myo}}$. At $t=30 \mathrm{~s}$, SERCA inhibition by thapsigargin (TG) is simulated by setting uptake rate constant $v_{\text {pump }}=0$. 

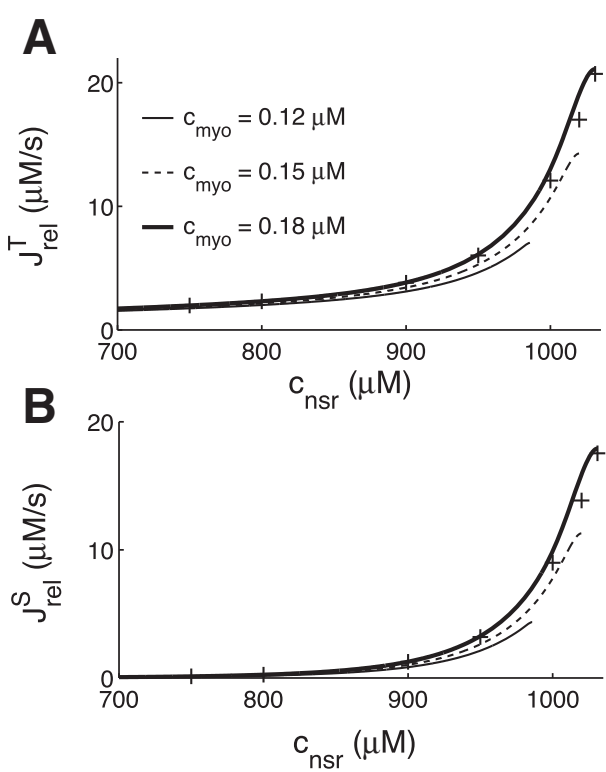
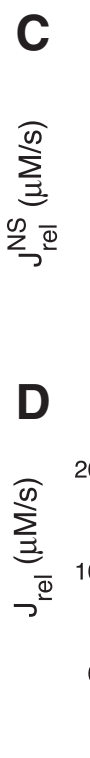
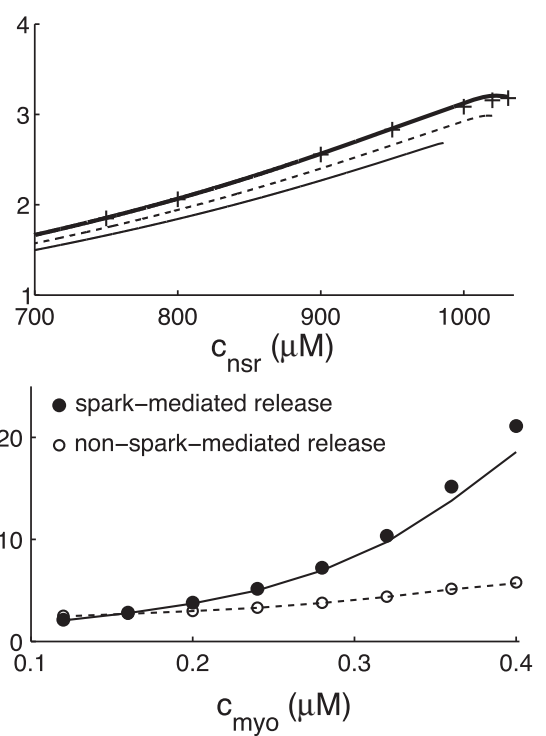

Fig. 7. Spark- and nonspark-mediated release during SR $\mathrm{Ca}^{2+}$ depletion. Total $\left(J_{\text {rel }}^{\mathrm{T}} ; A\right)$, spark mediated $\left(J_{\text {rel }}^{\mathrm{S}} ; B\right)$, and nonspark mediated $\left(J_{\text {rel }}^{\mathrm{NS}}\right.$; $C$ ) release flux are shown as functions of network SR $\left[\mathrm{Ca}^{2+}\right], \mathrm{c}_{\mathrm{nsr}}$, during SR depletion simulations (see Fig. 6), for different values of myoplasmic $\left[\mathrm{Ca}^{2+}\right], \mathrm{c}_{\text {myo. }}$. Steady-state calculations of $J_{\text {rel }}^{\mathrm{T}}, J_{\text {rel }}^{\mathrm{S}}$, and $J_{\text {rel }}^{\mathrm{NS}}$ ("+" symbols) are shown for $c_{\text {myo }}=0.18 \mu \mathrm{M} . D: J_{\text {rel }}^{\mathrm{S}}$ (filled circles) and $J_{\mathrm{rel}}^{\mathrm{NS}}$ (open circles) as functions of $\mathrm{c}_{\mathrm{myo}}$ for $\mathrm{c}_{\mathrm{nsr}}=950 \mu \mathrm{M}$. Solid and dash lines indicate steady-state $J_{\text {rel }}^{\mathrm{S}}$ and $J_{\text {rel }}^{\mathrm{NS}}$, respectively. and nonspark-mediated $\mathrm{SR} \mathrm{Ca}^{2+}$ release. However, for a slightly larger value of $\mathrm{c}_{\mathrm{nsr}}=1,020 \mu \mathrm{M}$, larger $N$ decreases nonspark-mediated release $\left(J_{\text {rel }}^{\mathrm{NS}}\right)$ while promoting robust sparks and increasing spark-mediated release $J_{\text {rel }}^{\mathrm{S}}$. However, if the total release flux $\left(v_{\text {rel }}^{\mathrm{T}}\right)$ is proportional to $N$ (as opposed to a constant), larger $N$ results in higher release flux regardless of $\mathrm{c}_{\mathrm{nsr}}$ because of high release flux rate (see APPENDIX E).

Finally, Fig. 10 shows the steady-state spark Score for "clamped" $c_{\text {myo }}$ and $c_{n s r}$ and illustrates the interplay of bulk concentrations and $\mathrm{Ca}^{2+}$ sparks. For a given value of $\mathrm{c}_{\text {myo }}$, the Score is a bell-shaped function of $\mathrm{c}_{\mathrm{nsr}}$, that is, there is a specific range of $\mathrm{SR} \mathrm{Ca}^{2+}$ load that supports robust sparks. As observed in prior work (21), the range for robust sparks decreases as $N$ is increased (Fig. 10, $B$ and $C$ ). Most importantly, the solid black lines indicate the steady-state (unclamped) network SR $\left[\mathrm{Ca}^{2+}\right]\left(\mathrm{c}_{\mathrm{nsr}}\right)$ as a function of $\mathrm{c}_{\text {myo }}$ (cf. Fig. $4 B$ ). When $\mathrm{c}_{\text {myo }}$ is sufficiently elevated that further increase leads to decreased $\mathrm{c}_{\mathrm{nsr}}$, the $\mathrm{SR} \mathrm{Ca}^{2+}$ load equilibrates to a value that maximizes the Score, that is, the steady-state $\mathrm{c}_{\mathrm{nsr}}$ decreases (with increasing $\mathrm{c}_{\mathrm{myo}}$ ) just enough to maintain robust sparks. This intriguing and potentially significant result is also observed when the total release flux $v_{\text {rel }}^{T}$ is proportional to $N$ (not shown).

\section{DISCUSSION}

Summary of main findings. In this article, we present a novel local/global whole cell model of $\mathrm{Ca}^{2+}$ homeostasis based on a Langevin description of stochastic $\mathrm{Ca}^{2+}$ release that includes both spark-mediated and nonspark-mediated release dynamics. The Fokker-Planck equation associated with the Langevin formulation of stochastic $\mathrm{Ca}^{2+}$ release is coupled to balance equations for the bulk myoplasmic and network SR $\left[\mathrm{Ca}^{2+}\right]$. With the use of this approximate representation of the collective dynamics of a large number of identical CaRUs, this whole cell modeling approach avoids Monte Carlo simulation of a large population of CaRUs and facilitates our study of $\mathrm{Ca}^{2+}$ homeostasis in permeabilized ventricular myocytes.

In permeabilized myocytes, the interplay between bulk myoplasmic $\left[\mathrm{Ca}^{2+}\right]\left(\mathrm{c}_{\text {myo }}\right)$, and network SR $\left[\mathrm{Ca}^{2+}\right]\left(\mathrm{c}_{\mathrm{nsr}}\right)$ on SR $\mathrm{Ca}^{2+}$ release is complex, in spite of the fact that myoplasmic
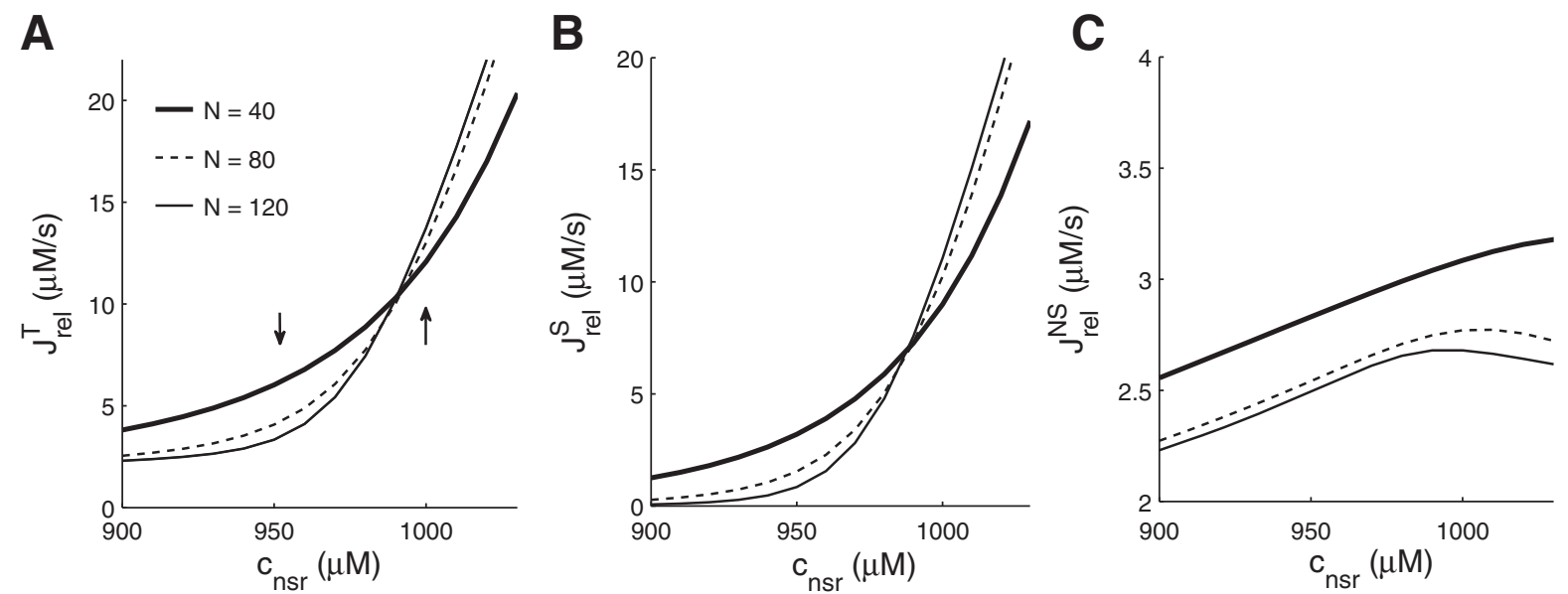

Fig. 8. Total $(A)$, spark-mediated $(B)$, and nonspark-mediated $(C)$ release flux as functions of $\mathrm{c}_{\mathrm{nsr}}$ at steady state. Parameters: $N=40$ (thick line), 80 (dash line), and 120 (thin line); $\mathrm{c}_{\mathrm{myo}}=0.18 \mu \mathrm{M}$; other parameters as in Table 1 . 
Fig. 9. Steady-state density of the fraction of open channels and release flux density for $\mathrm{c}_{\mathrm{nsr}}=950(A$ and $B)$ and $\mathrm{c}_{\mathrm{nsr}}=1,020 \mu \mathrm{M}(C$ and $D)$. Parameters: $N=40$ (thick line), 80 (dash line), and 120 (thin line); $\mathrm{c}_{\mathrm{myo}}=$ $0.18 \mu \mathrm{M}$; other parameters as in Table 1 .
A
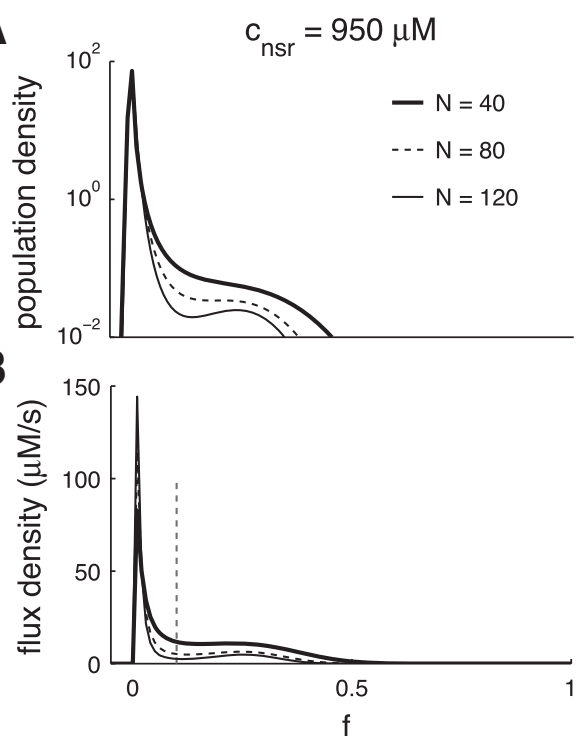

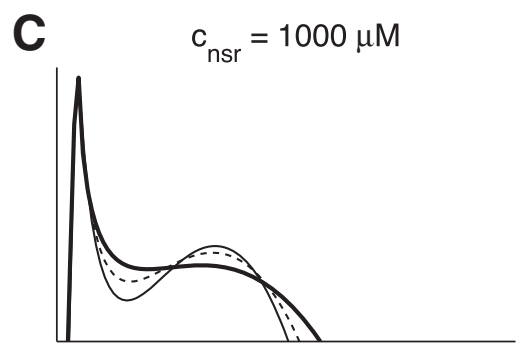

D

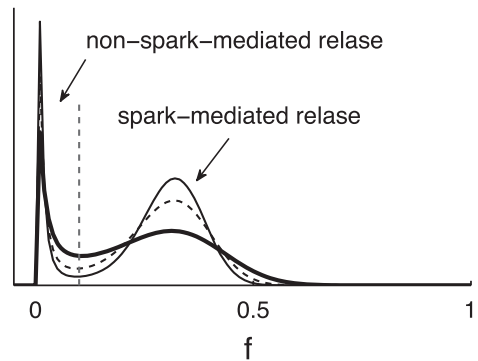

$\left[\mathrm{Ca}^{2+}\right]$ is under experimental control (i.e., $\mathrm{c}_{\mathrm{myo}}$ is not a dynamic variable but a model parameter). Elevated $\mathrm{c}_{\text {myo }}$ promotes $\mathrm{Ca}^{2+}$ uptake into the network SR via the SERCA pump, and this may elevate $\mathrm{c}_{\mathrm{nsr}}$. On the other hand, high $\mathrm{c}_{\mathrm{myo}}$ and high $\mathrm{c}_{\mathrm{nsr}}$ both promote increased SR $\mathrm{Ca}^{2+}$ release and depletion of $\mathrm{SR} \mathrm{Ca}^{2+}$.

We use the Langevin and Fokker-Planck local/global whole cell model of a permeabilized ventricular myocyte to characterize the depletion of network SR $\left[\mathrm{Ca}^{2+}\right]\left(\mathrm{c}_{\mathrm{nsr}}\right)$ that occurs via both spark-mediated release and nonspark-mediated release, as well as dependency of $\mathrm{SR} \mathrm{Ca}^{2+}$ load on myoplasmic $\left[\mathrm{Ca}^{2+}\right]$ $\left(c_{\text {myo }}\right)$. In agreement with recent experimental work (3), we find that spark-mediated release increases exponentially as $\mathrm{c}_{\mathrm{myo}}$ increases, while nonspark-mediated release increases linearly (Fig. 7).

The interplay among $\mathrm{c}_{\mathrm{myo}}, \mathrm{c}_{\mathrm{nsr}}$, and spark- and nonsparkmediated release in the local/global whole cell model generates several phenomena of $\mathrm{Ca}^{2+}$ homeostasis in permeabilized cells that are worth highlighting. For example, the model predicts the presence of two distinct stable steady states that lead to the same SR $\mathrm{Ca}^{2+}$ load, one with low myoplasmic $\left[\mathrm{Ca}^{2+}\right]$ and predominantly nonspark-mediated $\mathrm{SR} \mathrm{Ca}^{2+}$ release and another with high myoplasmic $\left[\mathrm{Ca}^{2+}\right]$ and release that is primarily spark mediated (Fig. 5). Significantly, in our permeabilized ventricular myocyte model, for any clamped myoplasmic $\left[\mathrm{Ca}^{2+}\right]\left(\mathrm{c}_{\mathrm{myo}}\right)$ that is large enough to trigger spark-mediated release, the resulting spontaneous stochastic $\mathrm{Ca}^{2+}$ release tends to decrease the network SR $\mathrm{Ca}^{2+}$ load just enough to maintain robust $\mathrm{Ca}^{2+}$ sparks (Fig. 10). To our knowledge this potentially significant characteristic of $\mathrm{Ca}^{2+}$ homeostasis in permeabilized cells has not previously been identified.

Physiological significance. Significant effort in recent years has been devoted to understanding the mechanisms influencing RyR regulation and $\mathrm{SR} \mathrm{Ca}^{2+}$ release. Abnormal regulation of RyRs can lead to aberrant SR $\mathrm{Ca}^{2+}$ release that directly contributes to excitation-contraction coupling dysfunction (13, 14). Previous studies have shown RyR-mediated $\mathrm{Ca}^{2+}$ release was enhanced in myocytes from failing rabbit hearts (40), which increases the likelihood of $\mathrm{Ca}^{2+}$-dependent arrhythmias (13). Recent experiments suggested that hidden RyR release contributes to the total release flux and influences $\mathrm{Ca}^{2+}$ homeostasis $(3,4,40)$. In this article, we are particularly inter-
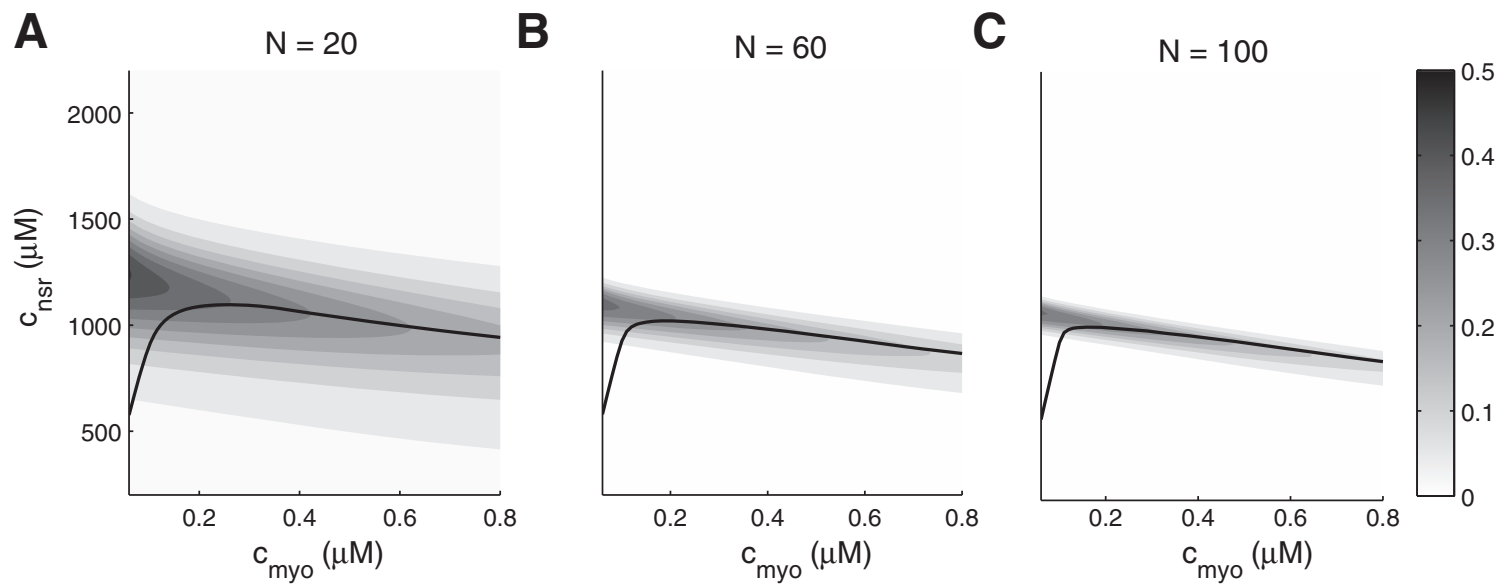

Fig. 10. Score as function of $\mathrm{c}_{\mathrm{myo}}$ and $\mathrm{c}_{\mathrm{nsr}}$ when $N$ is $20(A), 60(B)$, and $100(C)$, respectively. The solid line indicates the steady-state value for $\mathrm{c}_{\mathrm{nsr}}$ as a function of $\mathrm{c}_{\text {myo }}$ (cf. Fig. 4). 
ested in how myoplasmic $\left[\mathrm{Ca}^{2+}\right]\left(\mathrm{c}_{\mathrm{myo}}\right)$ influences $\mathrm{SR}^{2+}$ release via regulation of stochastic $\mathrm{Ca}^{2+}$ release mediated by CaRUs composed of clusters of RyRs. Our model shows that RyRs may produce both visible (spark-mediated) and invisible (nonspark-mediated) stochastic $\mathrm{Ca}^{2+}$ release. High $\mathrm{c}_{\mathrm{myo}}$ increases both spark- and nonspark-mediated release by increasing the open probability of $\mathrm{Ca}^{2+}$-activated RyRs. However, $\mathrm{c}_{\mathrm{myo}}$ affects these pathways in two distinct and characteristic ways. Nonspark-mediated $\mathrm{Ca}^{2+}$ release increases linearly as a function of $\mathrm{c}_{\mathrm{myo}}$, while spark-mediated release increases exponentially with $\mathrm{c}_{\mathrm{myo}}$.

We investigated how the number of RyRs in each individual CaRU influences network SR $\mathrm{Ca}^{2+}$ depletion and stochastic $\mathrm{Ca}^{2+}$ release. When $v_{\text {rel }}^{\mathrm{T}}$ is fixed (single channel conductance inversely proportional to $N$ ), we found that a larger number of RyRs per CaRU results in a steeper release flux (primarily spark-mediated release) as a function of network SR $\left[\mathrm{Ca}^{2+}\right]$, when the SR is replete. However, when network SR $\left[\mathrm{Ca}^{2+}\right]$ is depleted, and the release flux is primarily nonspark mediated, increasing the number of RyRs per CaRU decreases the total release flux, due to reduced triggering of $\mathrm{Ca}^{2+}$ sparks (Figs. 8 and 9). When $v_{\text {rel }}^{\mathrm{T}}$ is proportional to $N$ (fixed single channel conductance), $\mathrm{SR} \mathrm{Ca}^{2+}$ decreases with increasing $N$, due to higher release rates (not shown).

Because recent studies have shown that the number of RyRs per CaRU is variable (1), we note that the local/global whole cell model presented here can be modified to account for CaRUs of different size by simultaneously solving multiple Fokker-Planck equations, each with a different value for $N$. Assuming $M=\sum_{i} M_{i}$ CaRUs, with CaRUs of type $i$ composed of $N_{i}$ RyRs, the population densities $\rho_{i}$ solve

$$
\frac{\partial \rho_{i}}{\partial t}=-\frac{\partial}{\partial f}\left[\alpha_{i} \rho_{i}\right]+\frac{1}{2} \frac{\partial^{2}}{\partial f^{2}}\left[\gamma_{i} \rho_{i}\right]
$$

where $\alpha_{i}=v_{i}^{+}-v^{-}, \gamma_{i}=\left(v_{i}^{+}+v_{i}^{-}\right) / N, v_{i}^{+}=k^{+}\left(\bar{c}_{\mathrm{ds}}^{i}\right)^{\eta}(1-$ f) and $v^{-}=k^{-}$f. The stochastic $\mathrm{Ca}^{2+}$ release flux (Eq. 27) becomes

$$
\begin{aligned}
J_{\mathrm{nsr}}^{\mathrm{T}}(t) & =\frac{1}{M} \sum_{i} M_{i} \int v_{\mathrm{nsr}}^{\mathrm{T}}\left(\mathrm{c}_{\mathrm{nsr}}-\overline{\mathrm{c}}_{\mathrm{jsr}}^{i}\right) \rho_{i}(f, t) d f \\
& =v_{\mathrm{nsr}}^{\mathrm{T}}\left(\mathrm{c}_{\mathrm{nsr}}-\frac{1}{M} \sum_{i} M_{i} \int \overline{\mathrm{c}}_{\mathrm{jsr}}^{i} \rho_{i}(f, t) d f\right)
\end{aligned}
$$

where $\int \rho_{i} \mathrm{~d} f=1$ and thus $M^{-1} \sum_{i} \int M_{i} \rho_{i} \mathrm{~d} f=1$. In these equations, $\overline{\mathrm{c}}_{\mathrm{ds}}^{i}(f)$ and $\overline{\mathrm{c}}_{\mathrm{jsr}}^{i}(f)$ are given by indexed versions of Eqs. 32 and 33 where $\widetilde{v}_{\text {rel }}^{i}=v_{\text {rel }}^{0} N_{i} f$ and $v_{\text {rel }}^{0}$ is analogous to the RyR unitary conductance. Writing $v_{\text {myo }}^{i}$ and $v_{\mathrm{nsr}}^{i}$ as the domain time constants for a representative of the $i$ th class of CaRU, $\chi_{\text {myo }}^{i}$ and $\chi_{\mathrm{nsr}}^{i}$ are given by Eqs. 34-37 upon replacement of $i$ for T. The Fokker-Planck equations are coupled, because $\alpha_{i}$ is a function of $\mathrm{c}_{\mathrm{nsr}}$ through $\overline{\mathrm{c}}_{\mathrm{ds}}^{i}$, and $\mathrm{dc}_{\mathrm{nsr}} / \mathrm{d} t$ depends on the $\rho_{i}$ through $v_{\text {nsr }}^{\mathrm{T}}(E q .32)$.

Comparison to other whole cell models. A number of mathematical and computational whole cell models have been developed to understand $\mathrm{Ca}^{2+}$ homeostasis and the cardiac $\mathrm{Ca}^{2+}$ cycle. For example, computational models of excitationcontraction coupling in ventricular myocytes have been developed in which SR $\mathrm{Ca}^{2+}$ release depends directly on the average myoplasmic $\left[\mathrm{Ca}^{2+}\right](25,34)$. These "common pool" models (33) exhibit all-or-none triggered $\mathrm{SR} \mathrm{Ca}^{2+}$ release, contrary to experiments showing that release is smoothly graded with changes in $\mathrm{Ca}^{2+}$ influx $(5,36)$. This discrepancy is a consequence of the "local control" mechanism of CICR. In ventricular myocytes, the cellular SR $\mathrm{Ca}^{2+}$ release flux is not a function of the spatially averaged intracellular $\left[\mathrm{Ca}^{2+}\right]$ but instead depends on thousands of different local $\mathrm{Ca}^{2+}$ concentrations fluctuating in response to stochastic openings and closings of RyRs located on the SR membrane. The picture is further complicated by dynamic changes in localized SR $\left[\mathrm{Ca}^{2+}\right]$ that are also spatially heterogeneous and thought to influence the gating of RyRs (31).

To overcome this problem, stochastic models that account for the heterogeneous dyadic subspace and junctional SR $\left[\mathrm{Ca}^{2+}\right]$ have been developed $(19,22,39)$. Similar to the Langevin model that is the focus of this article, these local control models include a large number of CaRUs. In such models, RyR stochastic gating is typically described by a discrete-state Markov chain. This approach has recently been used to examine issues such as allosteric coupling between RyRs (39) and refractoriness of $\mathrm{Ca}^{2+}$ release after termination (28).

While Markov chain and Langevin models of CaRUs may lead to similar results (Fig. 1), the state space for Markov chain simulations is proportional to the number of $\mathrm{CaRU}$ states, a quantity that is exponential in the number of distinct RyR states. To see this, consider a CaRU composed $N$ identical $K$-state channels (and thus $K^{N}$ states). It is well-known that the number of distinguishable CaRU states is given by $(N+K-$ $1) ! / N ! /(K-1) !=[(N+K-1) \ldots(N+1)] /(K-1) !$, a quantity that includes a term proportional to $N^{K-1}$ (the numerator has $K-1$ terms) and is thus exponential in $K$. On the other hand, the run time for Langevin simulations is independent of the number of RyRs ( $N$ is a model parameter that scales the channel noise) and proportional to the number of RyR states $K$ (the required number of SDEs). Similarly, the run time of the Langevin local/global model does not scale with $N$, and the model may be extended to include RyRs with more than two states (see below). Because the Langevin version of the local/ global model that has been our focus involves only a single SDE (two-state RyR model), the probability density function for CaRU state is univariate. For this reason, the Fokker-Planck local/global whole cell model is extremely computationally efficient. Because a $K$-state RyR model leads to a FokkerPlanck equation with $K-1$ independent variables (conservation of probability), the Langevin version of the local/global model is likely to be more straightforward than the FokkerPlanck version when $K \geq 3$ (see $E q$. 41 below).

It is instructive to compare the local/global model presented here with our prior work. In Hartman et al. (22), we presented a similar minimal model of a permeabilized myocyte, in which bulk myoplasmic and network SR $\mathrm{Ca}^{2+}$ levels were coupled to a Markov chain CaRU model with $\mathrm{NCa}^{2+}$-activated RyRs per release site. The master equation in this case was a linear system of $N+1$ ODEs. The Langevin and Fokker-Planck local/global models presented here are also distinct from prior work of Williams et al. $(37,38)$. In these studies, $\mathrm{Ca}^{2+}$ release dynamics were described by a set of coupled multivariate probability density functions (advection-reaction equations) for the dyadic subspace and junctional SR $\left[\mathrm{Ca}^{2+}\right], \mathrm{c}_{\mathrm{ds}}$ and $\mathrm{c}_{\mathrm{jsr}}$, conditioned on CaRU state. This population density method and the associated moment-based reductions (38) are limited 
by a state-space explosion that is exponential in $K$, while the computational efficiency of the Langevin local/global model is linear in $K$.

Limitations and extensions of the model. In the Langevin model, we assume that the number of channels in each CaRUs is large enough that the fraction of RyRs in different states can be treated as a continuous variable. When the number of RyRs per CaRU is small, the error associated with the Langevin approximation to the Markov chain CaRU model may not be acceptable (15). In the local/global whole cell model presented here, the Langevin formulation was validated using a physiologically realistic numbers of RyRs per CaRU (see Figs. B1 and B2). The number of RyRs per CaRU required for the Langevin formulation to be highly accurate likely depends on the details of the RyR model used but is easily determined in any specific case.

In the derivation of the reduced local/global model, we assume that the dynamics of dyadic subspace $\left[\mathrm{Ca}^{2+}\right]$ and junction SR $\left[\mathrm{Ca}^{2+}\right]$ are fast compared with the gating of RyRs. However, slow translocation of junctional $\mathrm{SR}\left[\mathrm{Ca}^{2+}\right]$ can be incorporated into the Langevin local/global whole cell model through the addition of an additional SDE (24). This extension might be important if the chosen RyR model includes luminal regulation, that is, transitions whose rate is a function of junctional SR $\left[\mathrm{Ca}^{2+}\right]$. Accounting for slow junctional SR dynamics would increase the dimensionality of the probability density function (Eq. 28) used in the corresponding FokkerPlanck whole cell model.

Upgrading the Langevin formulation of the local/global whole cell model to accommodate more complex RyR models is straightforward. A $K$-state RyR model leads to a linear system of $K$ SDEs,

$$
\frac{\mathrm{d} f}{\mathrm{~d} t}=\boldsymbol{f} Q+\boldsymbol{\xi}(t)
$$

where $\mathbf{f}=\left(f_{1}, f_{2}, \ldots, f_{K}\right)$ and $\boldsymbol{\xi}=\left(\xi_{1}, \xi_{2}, \ldots, \xi_{K}\right)$ are row vectors, $Q=\left(q_{i j}\right)$ is the RyR model's transition matrix (the Markov chain's infinitesimal generator), the random term is mean zero $(\langle\boldsymbol{\xi}(t)\rangle=\mathbf{0})$ with two-time covariance matrix,

$$
\left\langle\xi^{\mathrm{T}}(t) \xi\left(t^{\prime}\right)\right\rangle=\Gamma(f) \delta\left(t-t^{\prime}\right),
$$

where $\Gamma=\left(\gamma_{i j}\right), \gamma_{i j}=-\left(q_{i j} f_{i}+q_{j i} f_{j}\right) / N$ for $i \neq j$ and $\gamma_{i i}=$ $-\sum_{j \neq i} \gamma_{i j}$ (the $\gamma_{i i}$ are positive) (26). The corresponding FokkerPlanck equation for the $K$-state RyR is

$$
\begin{aligned}
\frac{\partial}{\partial t} \rho(f, t)=-\sum_{i=1}^{K} \frac{\partial}{\partial f_{i}}\left[(f Q)_{i} \rho(f, t)\right] & \\
& +\frac{1}{2} \sum_{i=1}^{K} \sum_{j=1}^{K} \frac{\partial^{2}}{\partial f_{i} \partial f_{i}}\left[\gamma_{i j} \rho(f, t)\right],
\end{aligned}
$$

where $(\mathbf{f Q})_{i}$ is the $i$ th element of the row vector $\mathbf{f Q}$.

The Langevin and Fokker-Planck formulations of the local/ global whole cell model presented here are not explicitly spatial. That is, a large population of CaRUs are assumed to influence one another indirectly via the spatially averaged bulk myoplasmic and network SR $\left[\mathrm{Ca}^{2+}\right]$ (the CaRUs are mean-field coupled). This form of the local/global model is not well-suited to investigate macrosparks and other explicitly spatial phenomena that might occur in permeabilized ventricular myocytes when myoplasmic $\left[\mathrm{Ca}^{2+}\right]$ is very high.
By partitioning (discretizing) the bulk myoplasm and SR into regions that interact via buffered $\mathrm{Ca}^{2+}$ diffusion, the formalism would allow for propagation of intercellular $\mathrm{Ca}^{2+}$ waves, subcellular alternans, and so on. Such extensions of the Langevin local/global model approach would be straightforward and robust. Extending the Fokker-Planck local/global model in this way require a discretization sufficiently coarse that number of CaRUs per subcompartment remains large.

\section{APPENDIX A: DERIVATION OF THE LANGEVIN CARU MODEL}

Assuming a time interval $\Delta t$ is small enough so that at most one event occurs in the interval $[t, t+\Delta t]$, a CaRU composed of $N$ two-state channels will undergo a $N_{\mathrm{O}} \rightarrow N_{\mathrm{O}}-1$, transition $\left(\Delta N_{\mathrm{O}}=\right.$ $-1)$ with probability $p^{-}=k^{-} N_{\mathrm{O}} \Delta t$, and a $N_{\mathrm{O}} \rightarrow N_{\mathrm{O}}+1$ transition $\left(\Delta N_{\mathrm{O}}=1\right)$ with probability $p^{+}=\left(N-N_{\mathrm{O}}\right) k^{+} \mathrm{c}_{N_{\mathrm{O}}}^{n} \Delta t$, where $\mathrm{c}_{N_{\mathrm{O}}}$ is the local $\left[\mathrm{Ca}^{2+}\right]$ for $N_{\mathrm{O}}$ open channels $(E q$. 3). By conservation, the probability that $\Delta N_{\mathrm{O}}=0$ is $p^{0}=1-k^{-} N_{\mathrm{O}} \Delta t-\left(N-N_{\mathrm{O}}\right) k^{+} \mathrm{c}_{N_{\mathrm{O}}}^{\eta} \Delta t$. Conditioning on the current state, the expected infinitesimal increment of the number of open channels $\left(\Delta N_{\mathrm{O}}\right)$ is thus

$$
\begin{aligned}
\lim _{\Delta t \rightarrow 0} \frac{1}{\Delta t} \mathrm{E}\left[\Delta N_{\mathrm{O}} \mid N_{\mathrm{O}}(t)\right]=\lim _{\Delta t \rightarrow 0} \frac{1}{\Delta t}\left[-1 \cdot p^{-}+0 \cdot p^{0}+1 \cdot p^{+}\right] \\
=\left(N-N_{\mathrm{O}}\right) k^{+} c_{N_{\mathrm{O}}}^{\eta}-k^{-} N_{\mathrm{O}} .
\end{aligned}
$$

The deterministic part of the right hand side of Eq. 5 is derived as the corresponding infinitesimal expected increment in $f_{\mathrm{O}}=N_{\mathrm{O}} / N$. Similarly, the infinitesimal variance of $\Delta N_{\mathrm{O}}$ is

$$
\begin{array}{r}
\lim _{\Delta t \rightarrow 0} \frac{1}{\Delta t} \mathrm{E}\left[\Delta N_{\mathrm{O}}^{2} \mid N_{\mathrm{O}}(t)\right]=\lim _{\Delta t \rightarrow 0} \frac{1}{\Delta t}\left[(-1)^{2} \cdot p^{-}+0^{2} \cdot p^{0}+1^{2} \cdot p^{+}\right] \\
=\left(N-N_{\mathrm{O}}\right) k^{+} c_{N_{\mathrm{O}}}^{\eta}+k^{-} N_{\mathrm{O}} .
\end{array}
$$

The function $\gamma\left(f_{\mathrm{O}}\right)$ that occurs in $E q .8$ is derived from this quantity using $\mathrm{E}\left[\Delta f_{\mathrm{O}}^{2} \mid f_{\mathrm{O}}(t)\right]=\mathrm{E}\left[\Delta N_{\mathrm{O}}^{2} \mid N_{\mathrm{O}}(t)\right] / N^{2}$.

\section{APPENDIX B: COMPARISON OF MARKOV CHAIN AND LANGEVIN CARU MODELS}

Figure B1A compares the spark Score calculated via the Langevin (+ symbols) and the Markov chain (lines) description of a CaRU composed of two-state channels. The Score is a biphasic function of the coupling strength $\mathrm{c}_{*}(E q .38)$, with robust sparks occurring over a wider range of coupling strength when $N=20$ vs. 60 (dashed and solid lines, respectively). The Langevin method agrees with the Markov chain result, but overestimates the Score slightly for $N=20$ and small $\mathrm{c}_{*}$ (parameter regimes with few channel openings). Figure B $1 B$ shows that the Score calculated via the stationary distribution of the Markov chain and the Fokker-Planck equation are in agreement.

The Langevin method is also applicable to more complex single channel models. For example, consider a three-state RyR that is activated as well as inactivated by $\mathrm{Ca}^{2+}$,

$$
\mathrm{C}(\text { closed }) \underset{k_{a}^{-}}{\stackrel{k_{a}^{+} \mathrm{c}^{\eta}}{\rightleftharpoons}} \mathrm{O}(\text { open }) \underset{k_{b}^{+}}{\stackrel{k_{b}^{+} \mathrm{c}^{\eta}}{\rightleftharpoons}} \mathrm{R}(\text { refractory }),
$$

where $\mathrm{c}$ is the local $\left[\mathrm{Ca}^{2+}\right], k_{a}^{+} \mathrm{c}^{\eta}, k_{a}^{-}, k_{b}^{+} \mathrm{c}^{\eta}$, and $k_{b}^{-}$are transition rates with units of time ${ }^{-1}, k_{a}^{+}$and $k_{b}^{+}$are association rate constants with units of conc ${ }^{-}$- time ${ }^{-1}$, and the cooperativity of $\mathrm{Ca}^{2+}$ binding $\eta$ is the same for activation and inactivation. The Langevin description of a CaRU composed of $N$ three-state channels (Eq. 42) is given by Eq. 39, where the fraction of channels in each state, $\mathbf{f}=\left(f_{\mathrm{C}}, f_{\mathrm{O}}, f_{\mathrm{R}}\right)$, is a row vector, $Q$ is the transition rate matrix, 


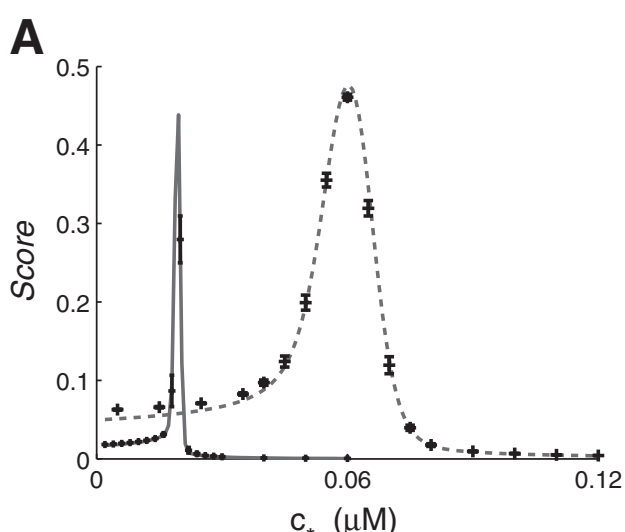

$$
Q=\left(q_{i j}\right)=\left(\begin{array}{ccc}
\diamond & k_{a}^{+} \mathrm{c}^{\eta} & 0 \\
k_{a}^{-} & \diamond & k_{b}^{+} \mathrm{c}^{\eta} \\
0 & k_{b}^{-} & \diamond
\end{array}\right),
$$

where the diagonal elements $(\diamond)$ are such that each row sums to zero $\left(\sum_{j} q_{\mathrm{ij}}=0\right)$ and the local $\left[\mathrm{Ca}^{2+}\right]$ is $\mathrm{c}=\mathrm{c}_{\infty}+f_{\mathrm{O}} \overline{\mathrm{c}}$ where $\overline{\mathrm{c}}=N \mathrm{c} *$ (cf. $E q .3)$. The rapidly varying forcing function in the Langevin equation (Eq. 39), $\boldsymbol{\xi}(t)=\left[\xi_{\mathrm{C}}(t), \xi_{\mathrm{O}}(t), \xi_{\mathrm{R}}(t)\right]$ is mean zero $(\langle\boldsymbol{\xi}(t)\rangle=\mathbf{0})$ with two-time covariance $\left\langle\boldsymbol{\xi}^{\mathrm{T}}(t) \boldsymbol{\xi}\left(t^{\prime}\right)\right\rangle=\Gamma(\mathbf{f}) \delta\left(t-t^{\prime}\right)(E q$. 40). Here $\Gamma=$ $\left(\gamma_{i j}\right)$ is given by $\gamma_{\mathrm{OC}}=\gamma_{\mathrm{CO}}=\left[k_{a}^{+} \mathrm{c}^{n} f_{\mathrm{C}}+k_{a}^{-} f_{\mathrm{O}}\right] / N, \gamma_{\mathrm{OR}}=\gamma_{\mathrm{RO}}=$ $\left[k_{b}^{+} \mathrm{c}^{\eta} f_{\mathrm{O}}+k_{b}^{-} f_{\mathrm{R}}\right] / N, \gamma_{\mathrm{CR}}=\gamma_{\mathrm{RC}}$ and the diagonal entries are such that each row sums to zero.

Figure B2 plots Score vs. coupling strength (c*) for this Langevin model of a CaRU composed of $N$ three-state channels with $\mathrm{Ca}^{2+}$ inactivation. This may be compared with the result for a $\mathrm{CaRU}$ composed of $N$ two-state channels with no inactivation (Fig. B1). Consistent with a previous computational study (21), Fig. B2 shows that $\mathrm{Ca}^{2}$-dependent inactivation facilitates spark termination (i.e., CaRUs spark for a wider range of coupling strengths). Most importantly, the Langevin (+ symbols) and Markov chain (lines) simulations agree.

\section{APPENDIX C: LANGEVIN EQUATION BOUNDARY CONDITIONS}

Because solutions of the Langevin CaRU model $\left(f_{\mathrm{i}}\right)$ represent the fraction of channels in state $i$, physical values are in the range $0 \leq f_{\mathrm{i}} \leq$ 1 and, formally, the stochastic processes that solve the Langevin CaRU models (Eqs. 5 and 39) have this property. However, numerical integration via the Euler-Maruyama method (16) involves a finite time step; consequently, there is a small probability of crossing $f_{i}=0$ or 1 , thereby exiting the physical range.

In the context of stochastic ODE modeling of ion channel dynamics, several modifications of the Euler-Maruyama scheme are commonly used to address this numerical issue. These include rejection and projection methods as well as more sophisticated approaches such as equilibrium noise approximations (18) and reflected stochastic differential equations (reviewed in Ref. 7). Unfortunately, these methods yield solutions that may disagree with the corresponding Markov chains when $N=20-200$ (11). In the context of Langevin CaRU models, a superior approach is to define auxiliary variables (observables) restricted to the physical range, i.e., $\hat{f}_{i}=\max \left[0, \min \left(1, f_{i}\right)\right]$, for evaluation of state-dependent rates, without projecting the stochastic trajectory $f_{i}$ to the boundary. For example, the Euler-Maruyama scheme use to integrate $E q .5$ is

$$
f^{m+1}=f^{m}+\Delta t\left[\hat{\alpha}\left(f^{m}\right)+\sqrt{\hat{\gamma}\left(f^{m}\right)} \Delta B^{m}\right]
$$

where the $\Delta B^{m}$ are i.i.d. normal random variables with mean zero and variance $1 / \Delta t, \hat{\alpha}(f)=k^{+} \hat{c}^{\eta}(1-f)-k^{-} f$, and $\hat{\gamma}(f)=\left[k^{+} \hat{c}^{\eta}(1-\right.$ $\left.\hat{f})+k^{-} \hat{f}\right] N$ and $\hat{c}=c_{\infty}+\bar{c} \hat{f}$. Because the deterministic flux is positive $(\alpha>0)$ when $f<0$ and negative $(\alpha<0)$ when $f>1$, no restriction is necessary for the factors $1-f$ and $f$ in $\hat{\alpha}$; in fact, we found that not doing so yields better agreement with the corresponding Markov chain simulations. Conversely, $\hat{f}$ is used in the evaluation of the diffusive term to ensure $\hat{\gamma}$. This method has similarities to the reflected stochastic differential equation technique discussed in Dangerfield et al. (2012).

\section{APPENDIX D: SPARK STATISTICS ANALYSIS VIA THE LANGEVIN DESCRIPTION OF THE LOCAL/GLOBAL WHOLE CELL MODEL}

Figure D1 shows the mean steady-state spark amplitude $(A)$, spark duration $(B)$, and interevent intervals $(C)$ as a function of $\mathrm{c}_{\mathrm{myo}}$, calculated via the Langevin version of the local/global whole cell model. The duration of the $i$ th $\mathrm{Ca}^{2+}$ release event is the time elapsed between the first channel opening and last channel closing of each simulated spark, here defined as $f_{\mathrm{O}}$ crossing the threshold

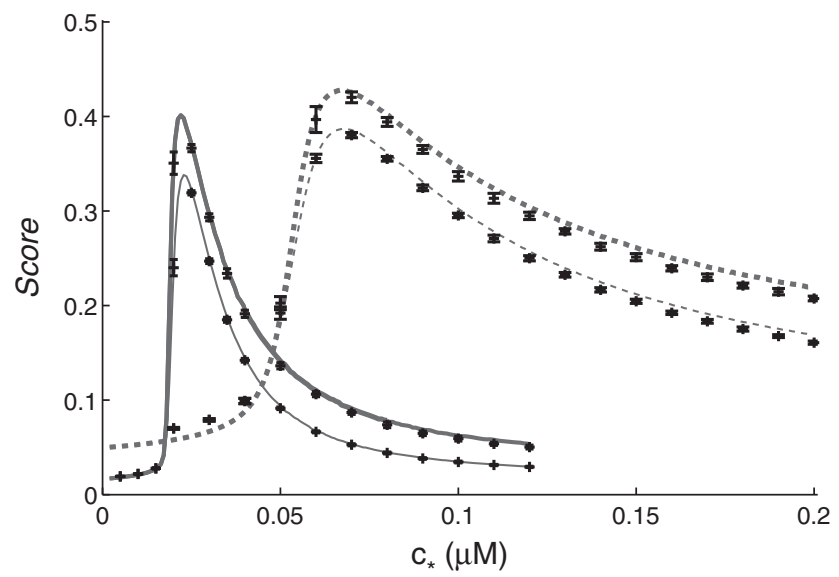

Fig. B2. Comparison of the Langevin (+ symbols) and the Markov chain (lines) description of an individual CaRU composed of $N=20$ (solid) or 60 (dashed lines) three-state channels that include fast $\mathrm{Ca}^{2+}$ activation and slow $\mathrm{Ca}^{2+}$ inactivation (Eq. 42). The dissociation constant of inactivating $\mathrm{Ca}^{2+}$ is fixed $\left(K_{b}=5.8\right)$; however, the thick lines indicate inactivation rates slowed 10 -fold compared with thin lines. Parameters: $c_{\infty}=0.05 \mu \mathrm{M}, \eta=2, k_{a}^{+}=1.5$ $\mu \mathrm{M}^{-\eta} \cdot \mathrm{ms}^{-1}, k_{a}^{-}=0.5 \mathrm{~ms}^{-1}$; thin lines: $k_{b}^{+}=0.015 \mu \mathrm{M}^{-\eta} \cdot \mathrm{ms}^{-1}, k_{b}^{-}=0.005$ $\mathrm{ms}^{-1}$; thick lines: $k_{b}^{+}=0.0015 \mu \mathrm{M}^{-\eta} \cdot \mathrm{ms}^{-1}, k_{b}^{-}=0.0005 \mathrm{~ms}^{-1}$. Error bars as in Fig. B1. 
A

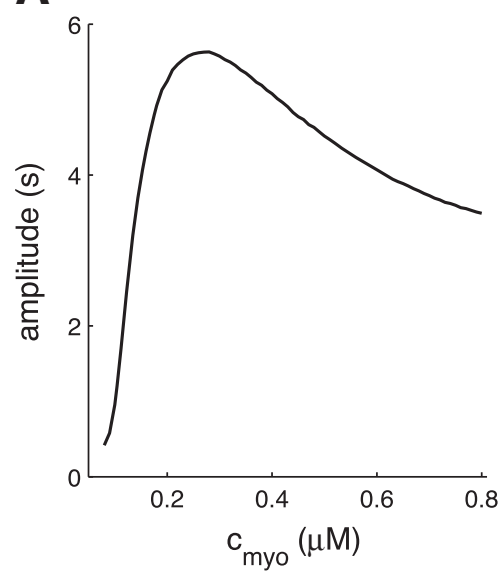

B

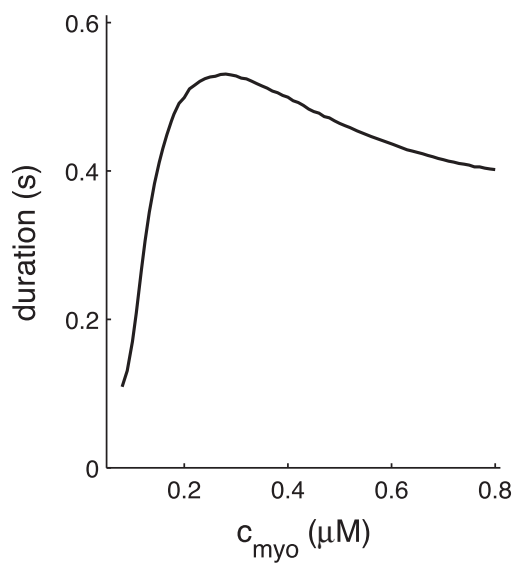

C

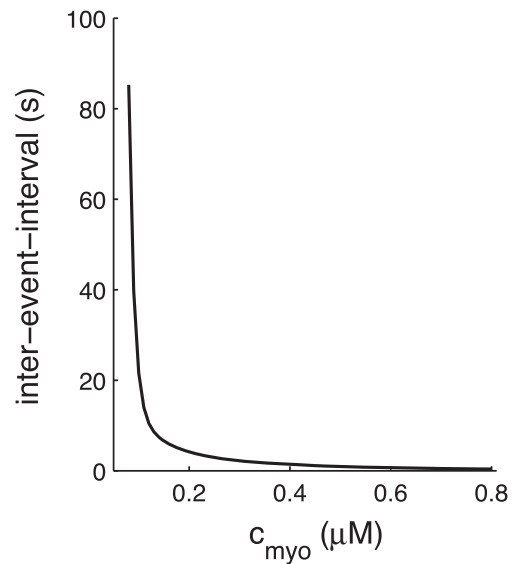

Fig. D1. Mean spark amplitude $(A)$, duration $(B)$, and interevent interval $(C)$ as a function of myoplasmic $\left[\mathrm{Ca}^{2+}\right]\left(\mathrm{c}_{\mathrm{myo}}\right)$, calculated via the Langevin version of the local/global whole cell model (average over 1,000-s simulations).

$1 / N$ in the upward/downward direction. The amplitude of $i$ th $\mathrm{Ca}^{2+}$ release event is the integrated area under $f_{\mathrm{O}}(t)$ during the event. The $i$ th interevent interval is the length of time between the $(i-$ 1)th and $i$ th $\mathrm{Ca}^{2+}$ release events. Note that spark amplitude and spark duration are biphasic functions of $\mathrm{c}_{\mathrm{myo}}$, peaking at $\mathrm{c}_{\mathrm{myo}} \approx$ $0.25 \mu \mathrm{M}$, similar to the steady-state $\mathrm{c}_{\mathrm{nsr}}$ and spark Score (Fig. $4, B$ and $C$ ).

\section{APPENDIX E: $\mathrm{CA}^{2+}$ RELEASE FLUX AND CARU SIZE}

Most of the parameter studies presented above assume that the total number of RyRs per cell is fixed. When the number of channels per CaRU $(N)$ is varied, the number of CaRUs per cell $(M)$ is changed so that $M N$ is a constant (i.e., the total release flux rate $v_{\text {rel }}^{\mathrm{T}}$ is fixed). Alternatively, $M$ may be fixed; in this case, $v_{\text {rel }}^{\mathrm{T}}$ is proportional to CaRU size $(N)$. Figure E1 shows the total release flux $\left(J_{\text {rel }}^{\mathrm{T}}\right)$, spark-mediated release $\left(J_{\text {rel }}^{\mathrm{S}}\right)$, and nonspark-mediated release $\left(J_{\text {rel }}^{\mathrm{NS}}\right)$ when the number of channels per $\mathrm{CaRU}(N)$ are varied under this assumption (fixed single channel conductance). In this case, regardless of $\mathrm{c}_{\mathrm{nsr}}$, the total release flux and spark-mediated release are higher for larger $N$. Conversely, when $v_{\text {rel }}^{\mathrm{T}}$ is fixed (Fig. 8), the clamped network SR $\left[\mathrm{Ca}^{2+}\right] \mathrm{c}_{\mathrm{nsr}}$ determines whether CaRU size $N$ increases or decreases the total release flux $J_{\text {rel }}^{\mathrm{T}}$. Figure E2 shows release flux density increases with CaRU size when $v_{\text {rel }}^{\mathrm{T}}$ is proportional to $N$ (cf. Fig. 9).

\section{GRANTS}

The work was supported in part by National Science Foundation Grant DMS 1121606 (to G. D. Smith) and the Biomathematics Initiative at The College of William \& Mary.

\section{DISCLOSURES}

No conflicts of interest, financial or otherwise, are declared by the author(s).

\section{AUTHOR CONTRIBUTIONS}

Author contributions: X.W., S.H.W., E.A.S., and G.D.S. conception and design of research; X.W. performed experiments; X.W. analyzed data; X.W., S.H.W., and G.D.S. interpreted results of experiments; X.W. prepared figures; X.W. drafted manuscript; X.W., S.H.W., Y.H., E.A.S., and G.D.S. edited and revised manuscript; X.W., S.H.W., Y.H., E.A.S., and G.D.S. approved final version of manuscript.

\section{REFERENCES}

1. Baddeley D, Jayasinghe ID, Lam L, Rossberger S, Cannell MB, Soeller C. Optical single-channel resolution imaging of the ryanodine
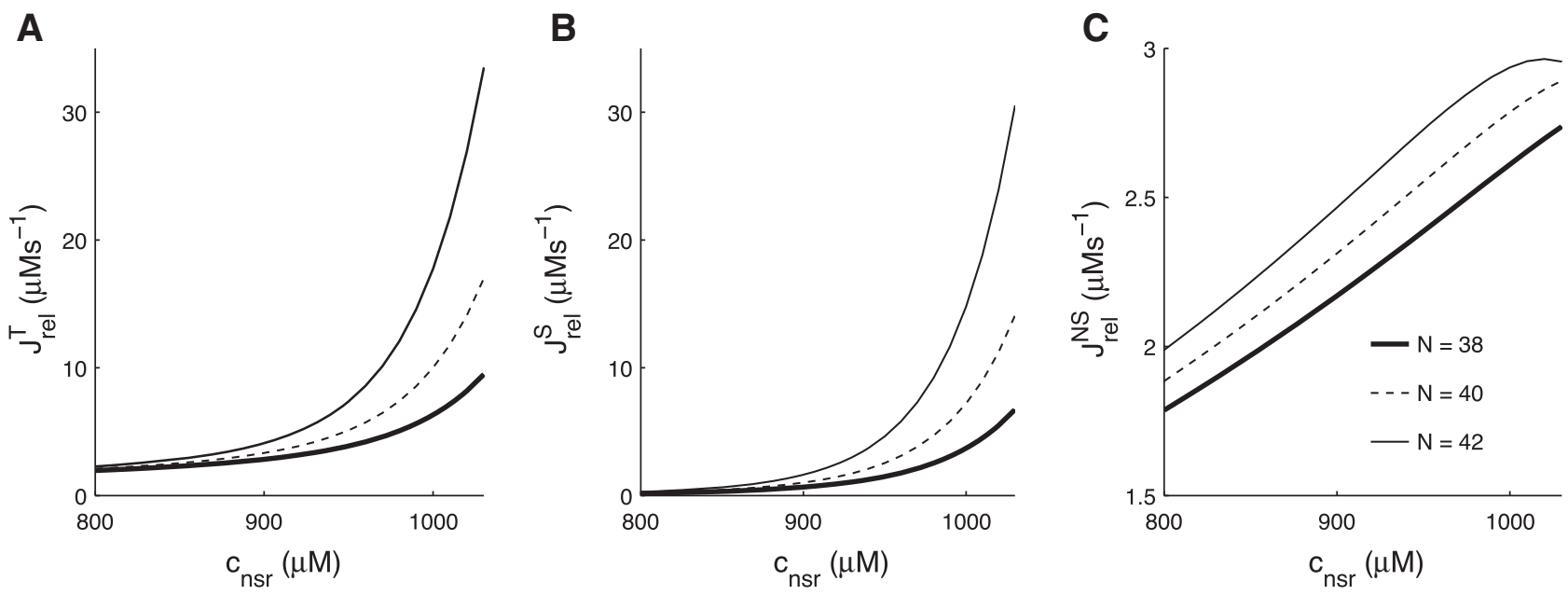

Fig. E1. Total $(A)$, spark-mediated $(B)$, and nonspark-mediated $(C)$ release flux as functions of $\mathrm{c}_{\mathrm{nsr}}$, calculated under the assumption that $v_{\mathrm{rel}}^{\mathrm{T}}$ is proportional to $N=38$ (thick line), 40 (dash line), and 42 (thin line) $\left(v_{\text {rel }}^{\mathrm{T}}=1.48,1.56\right.$, and $1.63 \mathrm{~s}^{-1}$, respectively). Other parameters: $\mathrm{c}_{\mathrm{myo}}=0.18 \mu \mathrm{M}$ and as in Table 1 . 

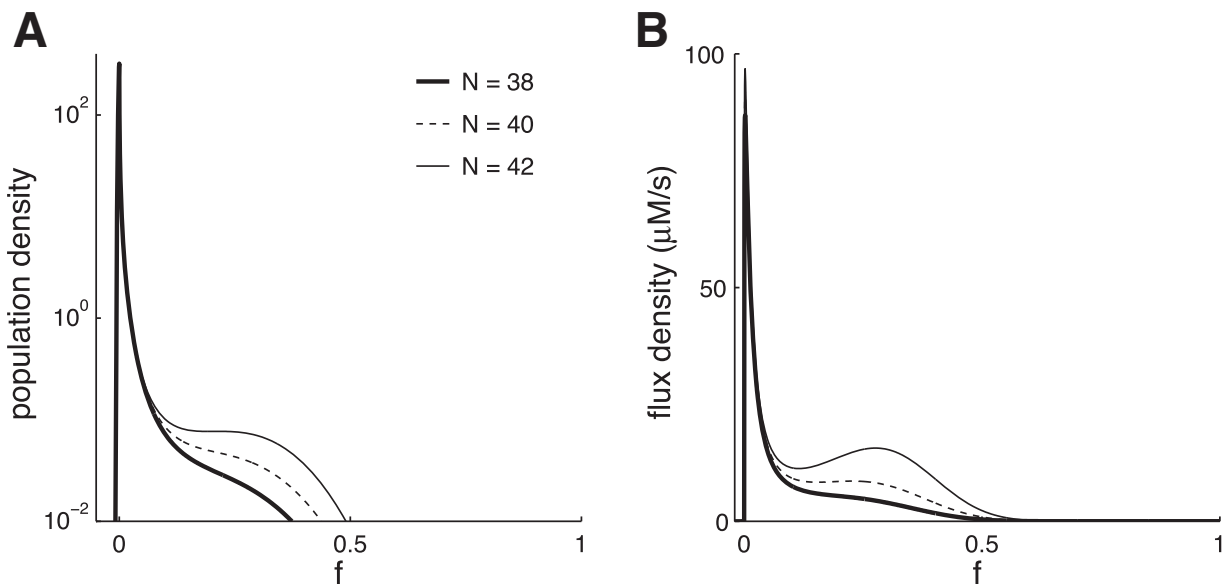

Fig. E2. Probability density function for the fraction of open channels $\rho_{\mathrm{ss}}(f)(A)$ and the release flux density $(B)$ for $\mathrm{c}_{\mathrm{nsr}}=950 \mu \mathrm{M}$ calculated under the assumption that $v_{\text {rel }}^{\mathrm{T}}$ is proportional to $N$. Parameters as in Fig. E1. receptor distribution in rat cardiac myocytes. Proc Natl Acad Sci USA 106: 22275-22280, 2009.

2. Berridge MJ. Calcium microdomains: organization and function. Cell Calcium 40: 405-412, 2006.

3. Bovo E, Mazurek SR, Blatter LA, Zima AV. Regulation of sarcoplasmic reticulum $\mathrm{Ca}^{2+}$ leak by cytosolic $\mathrm{Ca}^{2+}$ in rabbit ventricular myocytes. $J$ Physiol 589: 6039-6050, 2011.

4. Brochet DX, Xie W, Yang D, Chen H, Lederer WJ. Quarky calcium release in the heart. Circ Res 108: 210-218, 2011.

5. Cannell MB, Cheng H, Lederer WJ. The control of calcium release in heart muscle. Science 268: 1045-1049, 1995.

6. Chen-Izu Y, McCulle SL, Ward CW, Soeller C, Allen BM, Rabang C, Cannell MB, Balke CW, Izu LT. Three-dimensional distribution of ryanodine receptor clusters in cardiac myocytes. Biophys J 91: 1-13, 2006.

7. Dangerfield CE, Kay D, Burrage K. Modeling ion channel dynamics through reflected stochastic differential equation. Phys Rev E Stat Nonlin Soft Matter Phys 85: 051907, 2012.

8. DeRemigio H, Smith GD. The dynamics of stochastic attrition viewed as an absorption time on a terminating Markov chain. Cell Calcium 38: 73-86, 2005

9. Díaz ME, Trafford AW, O'Neill SC, Eisner DA. Measurement of sarcoplasmic reticulum $\mathrm{Ca}^{2+}$ content and sarcolemmal $\mathrm{Ca}^{2+}$ fluxes in isolated rat ventricular myocytes during spontaneous $\mathrm{Ca}^{2+}$ release. $J$ Physiol 501: 3-16, 1997.

10. Dibb K, Eisner D. A small leak may sink a great ship but what does it do to the heart? J Physiol 588: 4849, 2010.

11. Franzini-Armstrong C, Protasi F, Ramesh V. Shape, size, and distribution of $\mathrm{Ca}^{2+}$ release units and couplons in skeletal and cardiac muscles. Biophys J 77: 1528-1539, 1999.

12. Gardiner CW. Handbook of Stochastic Methods for Physics, Chemistry and the Natural Science. New York: Springer, 1985, p. 118-143.

13. George CH. Sarcoplasmic reticulum $\mathrm{Ca}^{2+}$ leak in heart failure: mere observation or functional relevance? Cardiovasc Res 77: 302-314, 2008.

14. George CH, Jundi H, Thomas NL, Fry DL, Lai FA. Ryanodine receptors and ventricular arrhythmias: emerging trends in mutations, mechanisms and therapies. J Mol Cell Cardiol 42: 34-50, 2007.

15. Gillespie DT. The chemical Langevin equation. J Chem Phys 113: 297-306, 2000.

16. Gillespie DT. Stochastic simulation of chemical kinetics. Апnи Rev Phys Chem 58: 35-55, 2007.

17. Gillespie DT. A general method for numerically simulating the stochastic time evolution of coupled chemical reactions. J Comp Physiol 22: 403 434, 1976

18. Goldwyn JH, Imennov NS, Famulare M, Shea-Brown E. Stochastic differential equation models for ion channel noise in Hodgkin-Huxley neurons. Phys Rev E Stat Nonlin Soft Matter Phys 83: 041908, 2011.

19. Greenstein JL, Winslow RL. An integrative model of the cardiac ventricular myocyte incorporating local control of $\mathrm{Ca}^{2+}$ release. Biophys J 83: 2918-2945, 2002.

20. Greenstein JL, Hinch R, Winslow RL. Mechanisms of excitationcontraction coupling in an integrative model of the cardiac ventricular myocyte. Biophys J 90: 77-91, 2006.

21. Groff JR, Smith GD. Calcium-dependent inactivation and the dynamics of calcium puffs and sparks. J Theor Biol 253: 483-499, 2008.
22. Hartman JA, Sobie EA, Smith GD. Calcium sparks and homeostasis in a minimal model of local and global calcium responses in quiescent ventricular myocytes. Am J Physiol Heart Circ Physiol 299: H1996-H2008, 2010.

23. Hinch R. A mathematical analysis of the generation and termination of calcium sparks. Biophys $J$ 86: 1293-1307, 2004.

24. Huertas MA, Smith GD, Györke S. Analysis of calcium alternans in a cardiac myocyte model that uses moment equations to represent heterogenous junctional SR calcium. Biophys $J$ 99: 377-387, 2010.

25. Jafri MS, Rice JJ, Winslow RL. Cardiac $\mathrm{Ca}^{2+}$ dynamics: the roles of ryanodine receptor adaptation and sarcoplasmic reticulum load. Biophys $J$ 74: 1149-1168, 1998.

26. Keizer JE. Statistical Thermodynamics of Nonequilibrium Processes. Berlin, Germany: Springer-Verlag, 1987.

27. Loughrey CM, MacEachern KE, Neary P, Smith GL. The relationship between intracellular $\left[\mathrm{Ca}^{2+}\right]$ and $\mathrm{Ca}^{2+}$ wave characteristics in permeabilised cardiomyocytes from the rabbit. J Physiol 543: 859-870, 2002.

28. Ramay HR, Liu OZ, Sobie EA. Recovery of cardiac calcium release is controlled by sarcoplasmic reticulum refilling and ryanodine receptor sensitivity. Cardiovas Res 91: 598-605, 2011.

29. Rice JJ, Jafri MS, Winslow RL. Modeling gain and gradedness of $\mathrm{Ca}^{2+}$ release in the functional unit of the cardiac diadic space. Biophys $J$ 77: $1871-1884,1999$.

30. Shannon JH, Ginsburg KS, Bers DM. Quantitative assessment of the SR $\mathrm{Ca}^{2+}$ leak-load relationship. Circ Res 91: 594-600, 2002.

31. Sobie EA, Lederer WJ. Dynamic local changes in sarcoplasmic reticulum calcium: physiological and pathophysiological roles. J Mol Cell Cardiol 52: 304-311, 2012.

32. Sobie EA, Dilly KW, dos Santos Cruz J, Lederer WJ, Jafri MS. Termination of cardiac $\mathrm{Ca}^{2+}$ sparks: an investigative mathematical model of calcium-induced calcium release. Biophys $J$ 83: 59-78, 2002.

33. Stern MD. Theory of excitation-contraction coupling in cardiac muscle. Biophys J 63: 497-517, 1992.

34. Tang Y, Othmer HG. A model of calcium dynamics in cardiac myocytes based on the kinetics of ryanodine-sensitive calcium channels. Biophys $J$ 67: 2223-2235, 1994.

35. Terentyev D, Viatchenko-Karpinski S, Valdivia HH, Escobar AL, Syörke S. Luminal $\mathrm{Ca}^{2+}$ controls termination and refractory behavior of $\mathrm{Ca}^{2+}$-induced $\mathrm{Ca}^{2+}$ release in cardiac myocytes. Circ Res 91: 414-420, 2002.

36. Wier WG, Egan TM, López-López JR, Balke CW. Local control of excitation contraction coupling in rat heart cells. $J$ Physiol 474: 463-471, 1994.

37. Williams GS, Huertas MA, Sobie EA, Jafri MS, Smith GD. A probability density approach to modeling local control of $\mathrm{Ca}^{2+}$-induced $\mathrm{Ca}^{2+}$ release in cardiac myocytes. Biophys J 92: 2311-2328, 2007.

38. Williams GS, Huertas MA, Sobie EA, Jafri MS, Smith GD. Moment closure for local control models of $\mathrm{Ca}^{2+}$-induced $\mathrm{Ca}^{2+}$ release in cardiac myocytes. Biophys J 95: 1689-1703, 2008.

39. Williams GS, Chikando AC, Tuan HTM, Sobie EA, Lederer WJ, Jafri MS. Dynamics of calcium sparks and calcium leak in the heart. Biophys $J$ 101: 1287-1296, 2011

40. Zima AV, Bovo E, Bers DM, Blatter LA. $\mathrm{Ca}^{2+}$ spark-dependent and -independent sarcoplasmic reticulum $\mathrm{Ca}^{2+}$ leak in normal and failing rabbit ventricular myocytes. $J$ Physiol 588: 4743-4757, 2010. 